\title{
A coupled system of fractional differential equations on the half-line
}

\section{Chengbo Zhai ${ }^{1 *}$ (D) and Jing Ren ${ }^{1}$}

\author{
"Correspondence: \\ cbzhai@sxu.edu.cn \\ 'School of Mathematical Sciences, \\ Shanxi University, Taiyuan, P.R. China
}

\begin{abstract} domain

$$
\begin{aligned}
& D^{\alpha} u(t)+\varphi\left(t, v(t), D^{\gamma_{1}} v(t)\right)=0, \quad t \in[0,+\infty), \alpha \in(2,3], \\
& D^{\beta} v(t)+\psi\left(t, u(t), D^{\gamma_{2}} u(t)\right)=0, \quad t \in[0,+\infty), \beta \in(2,3],
\end{aligned}
$$
\end{abstract}

In this paper, we consider a new fractional differential system on an unbounded

subject to the conditions

$$
\begin{array}{lll}
\left.\beta^{\beta-\alpha} u(t)\right|_{t=0}=0, & \left.D^{\alpha-2} u(t)\right|_{t=0}=\int_{0}^{h} g_{1}(s) u(s) d s, & D^{\alpha-1} u(+\infty)=M u(\xi)+a, \\
\left.\beta^{\beta-\beta} v(t)\right|_{t=0}=0, & \left.D^{\beta-2} v(t)\right|_{t=0}=\int_{0}^{h} g_{2}(s) v(s) d s, & D^{\beta-1} v(+\infty)=N v(\eta)+b .
\end{array}
$$

The nonlinear terms $\varphi$ and $\psi$ are dependent on the fractional derivative of lower order $\gamma_{i} \in(0,1), i=1,2$, which creates additional complexity to verify the existence of solutions. Moreover, a proper choice of Banach space allows the solutions to be defined on the half-line. From some standard fixed point theorems, sufficient conditions for the existence and uniqueness of solutions to boundary value problems are developed. Finally, the main result is applied to an illustrative example.

MSC: 34A08; 34B15; 34B40

Keywords: Fractional differential equations; Existence and uniqueness; Unbounded domain; Standard fixed point theorems; Boundary value problem

\section{Introduction}

Fractional calculus has recently evolved as an excellent tool for mathematical modeling owing to its widespread applications in the fields of engineering, physics, electrodynamics of complex medium, photoelasticity, etc; one can see [1-12] and the references cited therein. Meanwhile, relevant theory of fractional differential and integral equations has been established, and the research on fractional differential equations for boundary value problems is in a stage of rapid development.

Based on some kinds of analytical techniques, boundary value problems involving fractional differential equations attracted a considerable attention; see [13-33] and the references therein. It not only has promotional value and practical significance in medical

(c) The Author(s) 2019. This article is distributed under the terms of the Creative Commons Attribution 4.0 International License (http://creativecommons.org/licenses/by/4.0/), which permits unrestricted use, distribution, and reproduction in any medium, provided you give appropriate credit to the original author(s) and the source, provide a link to the Creative Commons license, and indicate if changes were made. 
image processing, seismic analysis, and large-scale climate research, but also has important research potential on numerical analysis.

Recently, the study of coupled systems involving fractional differential equations appeared in the literature $[4,9,10,17,18,32,33]$. Much of the work has been considered on finite intervals; however, a study of boundary value problems on unbounded domain is well under way. Wang, Ahmad, and Zhang [34] studied a coupled system of fractional differential equations with $m$-point fractional boundary conditions

$$
\left\{\begin{array}{l}
D^{p} u(t)+f(t, v(t))=0, \quad p \in(2,3), \\
D^{q} v(t)+g(t, u(t))=0, \quad q \in(2,3), \\
u(0)=u^{\prime}(0)=0, \quad D^{p-1} u(+\infty)=\sum_{i=1}^{m-2} \beta_{i} u\left(\xi_{i}\right), \\
v(0)=v^{\prime}(0)=0, \quad D^{q-1} v(+\infty)=\sum_{i=1}^{m-2} \gamma_{i} v\left(\xi_{i}\right),
\end{array}\right.
$$

where $t \in J=[0,+\infty), f, g \in C(J \times \mathbb{R}, \mathbb{R}), 0<\xi_{1}<\xi_{2}<\cdots<\xi_{m-2}<+\infty, \beta_{i}, \gamma_{i}>0$ such that $0<\sum_{i=1}^{m-2} \beta_{i} u\left(\xi_{i}\right)<\Gamma(p)$ and $0<\sum_{i=1}^{m-2} \gamma_{i} \nu\left(\xi_{i}\right)<\Gamma(q) . D^{p}, D^{q}$ denote the standard Riemann-Liouville fractional derivatives. By virtue of standard fixed point theorems, the authors discussed the existence and uniqueness of solutions.

In [35], the authors investigated a class of fractional differential equations on an infinite interval

$$
D_{0^{+}}^{\alpha} u(t)+f\left(t, u(t), D_{0^{+}}^{\alpha-1} u(t)\right)=0, \quad t \in(0,+\infty)
$$

with integral boundary conditions

$$
\left\{\begin{array}{l}
u(0)=0, \\
D_{0^{+}}^{\alpha-1} u(\infty)=\int_{0}^{\tau} g_{1}(s) u(s) d s+a, \\
D_{0^{+}}^{\alpha-2} u(0)=\int_{0}^{\tau} g_{2}(s) u(s) d s+b,
\end{array}\right.
$$

where $2<\alpha \leq 3, f: \mathbb{R}^{+} \times\left(\mathbb{R}^{+}\right)^{2} \rightarrow \mathbb{R}^{+}, f(t, u, v) \not \equiv 0$, and $f$ satisfies $L^{1}$-Carathéodory conditions. Existence results for positive solutions to the boundary value problem were obtained in three cases by using Krasnoselskii's fixed point theorem.

To our knowledge, some remarkable results on the existence and multiplicity of solutions for fractional differential equations have been discussed widely on finite intervals [14-33]. Instead, it is relatively rare for work to be done related to existence results on infinite intervals [34-47].

In [31], the authors discussed the existence and uniqueness of positive solutions for the fractional differential equation

$$
\left\{\begin{array}{l}
D^{\alpha} u(t)=f\left(t, u(t), D^{p} u(t)\right), \\
I^{3-\alpha} u(0)=D^{\alpha-2} u(0)=u(1)=0,
\end{array}\right.
$$

where $0<p<1,2<\alpha<3, t \in(0,1), D^{\alpha}$ is the standard Riemann-Liouville fractional derivative of order $\alpha$. By applying a nonlinear alternative of Leray-Schauder type and the Banach contraction theorem, the existence and uniqueness of solutions were obtained.

Motivated by the above papers, we are devoted to establishing some results on the existence and uniqueness of solutions for a new coupled system of nonlinear fractional dif- 
ferential equations

$$
\begin{cases}D^{\alpha} u(t)=-\varphi\left(t, v(t), D^{\gamma_{1}} v(t)\right), \quad \gamma_{1} \in(0,1), & \\ D^{\beta} v(t)=-\psi\left(t, u(t), D^{\gamma_{2}} u(t)\right), & \gamma_{2} \in(0,1), \\ I^{3-\alpha} u(0)=0, & D^{\alpha-2} u(0)=\int_{0}^{h} g_{1}(s) u(s) d s,\end{cases}
$$

where $2<\alpha, \beta \leq 3,0<\gamma_{i}<1, i=1,2, t \in J=[0,+\infty), M, N$ are real numbers with $0<M \xi^{\alpha-1}<\Gamma(\alpha), 0<N \eta^{\beta-1}<\Gamma(\beta), \xi, \eta, h>0$, parameters $a, b \in \mathbb{R}^{+}, g_{1}, g_{2} \in L^{1}[0, h]$ are nonnegative functions, $\varphi, \psi \in C(J \times \mathbb{R} \times \mathbb{R}, \mathbb{R})$ and $D^{\alpha}$, $D^{\beta}$ denote the fractional derivatives of Riemann-Liouville type of order $\alpha$ and $\beta$. Our conclusion is a natural expansion of the previous results in [31].

In this paper, the aim is to deal with the new coupled system of fractional differential equations on infinite intervals. Sufficient conditions for the existence and uniqueness of unbounded solutions for system (1.1) are obtained base upon Schauder's fixed point theorem and the Banach contraction theorem. Unlike previous works, the main difficulty of this paper is that we have to construct an appropriate Banach space, because the functions $\varphi, \psi$ contain the fractional derivatives.

\section{Preliminaries and auxiliary results}

For the convenience of the readers, we recall some useful definitions and lemmas.

Definition 2.1 ([1]) The fractional integral of Riemann-Liouville type of order $\alpha>0$ of a function $f$ is defined as

$$
\left(I^{\alpha} f\right)(t)=\frac{1}{\Gamma(\alpha)} \int_{0}^{t}(t-s)^{\alpha-1} f(s) d s, \quad \alpha>0,
$$

provided the integral exists.

Definition 2.2 ([1]) The fractional derivative of Riemann-Liouville type of order $\alpha>0$ of a function $f$ is given by

$$
\left(D^{\alpha} f\right)(t)=\left(D^{\lceil\alpha\rceil} I^{\lceil\alpha\rceil-\alpha} f\right)(t)=\frac{1}{\Gamma(n-\alpha)}\left(\frac{d}{d t}\right)^{n} \int_{0}^{t} \frac{f(s)}{(t-s)^{\alpha-n+1}} d s
$$

where $\lceil\alpha\rceil$ is the smallest integer greater than or equal to $\alpha$, provided that the right-hand side is pointwise defined on $(0,+\infty)$.

For further analysis, let

$$
\begin{aligned}
& \sigma_{1}=\frac{1}{\Gamma(\alpha)-M \xi^{\alpha-1}}, \quad \sigma_{2}=\frac{1}{\Gamma(\beta)-N \eta^{\beta-1}}, \\
& \omega_{1}=\int_{0}^{h} g_{1}(t) t^{\alpha-1} d t, \quad \omega_{2}=\int_{0}^{h} g_{2}(t) t^{\beta-1} d t, \\
& \delta_{1}=\int_{0}^{h}\left(1+t^{\alpha-1}\right) g_{1}(t) d t, \quad \delta_{2}=\int_{0}^{h}\left(1+t^{\beta-1}\right) g_{2}(t) d t,
\end{aligned}
$$




$$
\begin{aligned}
& T_{1}(t)=\frac{\sigma_{1} M \xi^{\alpha-2} t^{\alpha-1}+t^{\alpha-2}}{\Gamma(\alpha-1)}, \quad T_{2}(t)=\frac{\sigma_{2} N \eta^{\beta-2} t^{\beta-1}+t^{\beta-2}}{\Gamma(\beta-1)}, \\
& l_{1}=\frac{1+\sigma_{1} M \xi^{\alpha-2}}{\left(1-\mu_{1}\right) \Gamma(\alpha-1)}, \quad l_{2}=\frac{1+\sigma_{2} N \eta^{\beta-2}}{\left(1-\mu_{2}\right) \Gamma(\beta-1)} .
\end{aligned}
$$

In this paper, we always assume that $g_{i}:[0,+\infty) \rightarrow[0,+\infty)$ are continuous, and $\mu_{i}=$ $\int_{0}^{h} g_{i}(t) T_{i}(t) d t<1, i=1,2$.

Lemma 2.1 Assume that $f \in L^{1}(J)$ with $0<M \xi^{\alpha-1}<\Gamma(\alpha), \alpha \in(2,3]$. Then the fractional differential equation

$$
D^{\alpha} u(t)+f(t)=0, \quad t \in[0,+\infty)
$$

with

$$
\left\{\begin{array}{l}
I^{3-\alpha} u(0)=0 \\
D^{\alpha-2} u(0)=\int_{0}^{h} g_{1}(s) u(s) d s \\
D^{\alpha-1} u(+\infty)=M u(\xi)+a
\end{array}\right.
$$

has the solution

$$
u(t)=a \sigma_{1} t^{\alpha-1}+\frac{a \sigma_{1} \omega_{1} T_{1}(t)}{1-\mu_{1}}+\int_{0}^{+\infty} H(t, s) f(s) d s
$$

where

$$
\begin{aligned}
& H(t, s)=H_{1}(t, s)+\frac{T_{1}(t)}{1-\mu_{1}} \int_{0}^{h} H_{1}(\tau, s) g_{1}(\tau) d \tau, \\
& H_{1}(t, s)=\sigma_{1} M t^{\alpha-1} G_{1}(\xi, s)+G_{1}(t, s),
\end{aligned}
$$

and

$$
G_{1}(t, s)=\frac{1}{\Gamma(\alpha)} \begin{cases}t^{\alpha-1}-(t-s)^{\alpha-1}, & 0 \leq s \leq t<+\infty \\ t^{\alpha-1}, & 0 \leq t \leq s<+\infty\end{cases}
$$

Proof First, we can reduce the above problem to an equivalent integral equation

$$
u(t)=c_{1} t^{\alpha-1}+c_{2} t^{\alpha-2}+c_{3} t^{\alpha-3}-I^{\alpha} f(t)
$$

for some $c_{i} \in \mathbb{R}, i=1,2,3$. By the condition $\left.I^{3-\alpha} u(t)\right|_{t=0}=0$, we have

$$
\begin{aligned}
I^{3-\alpha} u(t) & =c_{1} I^{3-\alpha} t^{\alpha-1}+c_{2} I^{3-\alpha} t^{\alpha-2}+c_{3} I^{3-\alpha} t^{\alpha-3}-I^{3-\alpha} I^{\alpha} f(t) \\
& =c_{1} \frac{\Gamma(\alpha)}{\Gamma(3)} t^{2}+c_{2} \frac{\Gamma(\alpha-1)}{\Gamma(2)} t+c_{3} \frac{\Gamma(\alpha-2)}{\Gamma(1)}-I^{3} f(t),
\end{aligned}
$$


since $I^{3} f(t) \rightarrow 0$ as $t \rightarrow 0$, we must set $c_{3}=0$. On application of $D^{\alpha-2} u(0)=\int_{0}^{h} g_{1}(s) u(s) d s$ and $D^{\alpha-1} u(+\infty)=M u(\xi)+a$, we have

$$
\begin{aligned}
D^{\alpha-2} u(t) & =c_{1} D^{\alpha-2} t^{\alpha-1}+c_{2} D^{\alpha-2} t^{\alpha-2}-D^{\alpha-2} I^{\alpha} f(t) \\
& =c_{1} \Gamma(\alpha) t+c_{2} \Gamma(\alpha-1)-\int_{0}^{t}(t-s) f(s) d s, \\
D^{\alpha-1} u(t) & =c_{1} D^{\alpha-1} t^{\alpha-1}+c_{2} D^{\alpha-1} t^{\alpha-2}-D^{\alpha-1} I^{\alpha} f(t) \\
& =c_{1} \Gamma(\alpha)-\int_{0}^{t} f(s) d s,
\end{aligned}
$$

that is,

$$
\begin{aligned}
c_{1}= & \frac{1}{\Gamma(\alpha)-M \xi^{\alpha-1}}\left(a+\int_{0}^{+\infty} f(s) d s+\frac{M \xi^{\alpha-2}}{\Gamma(\alpha-1)} \int_{0}^{h} g_{1}(s) u(s) d s\right. \\
& \left.-\frac{M}{\Gamma(\alpha)} \int_{0}^{\xi}(\xi-s)^{\alpha-1} f(s) d s\right), \\
c_{2}= & \frac{1}{\Gamma(\alpha-1)} \int_{0}^{h} g_{1}(s) u(s) d s .
\end{aligned}
$$

This implies

$$
\begin{aligned}
u(t)= & \sigma_{1} t^{\alpha-1}\left(a+\int_{0}^{+\infty} f(s) d s+\frac{M \xi^{\alpha-2}}{\Gamma(\alpha-1)} \int_{0}^{h} g_{1}(s) u(s) d s\right. \\
& \left.-\frac{M}{\Gamma(\alpha)} \int_{0}^{\xi}(\xi-s)^{\alpha-1} f(s) d s\right) \\
& +\frac{t^{\alpha-2}}{\Gamma(\alpha-1)} \int_{0}^{h} g_{1}(s) u(s) d s-\frac{1}{\Gamma(\alpha)} \int_{0}^{t}(t-s)^{\alpha-1} f(s) d s \\
= & a \sigma_{1} t^{\alpha-1}+\frac{\sigma_{1} M \xi^{\alpha-2} t^{\alpha-1}+t^{\alpha-2}}{\Gamma(\alpha-1)} \int_{0}^{h} g_{1}(s) u(s) d s-\frac{\sigma_{1} M t^{\alpha-1}}{\Gamma(\alpha)} \int_{0}^{\xi}(\xi-s)^{\alpha-1} f(s) d s \\
& +\left(\frac{t^{\alpha-1}}{\Gamma(\alpha)}+\frac{\sigma_{1} M \xi^{\alpha-1} t^{\alpha-1}}{\Gamma(\alpha)}\right) \int_{0}^{+\infty} f(s) d s-\frac{1}{\Gamma(\alpha)} \int_{0}^{t}(t-s)^{\alpha-1} f(s) d s \\
= & a \sigma_{1} t^{\alpha-1}+\frac{\sigma_{1} M \xi^{\alpha-1} t^{\alpha-1}}{\Gamma(\alpha)} \int_{0}^{+\infty} f(s) d s-\frac{\sigma_{1} M t^{\alpha-1}}{\Gamma(\alpha)} \int_{0}^{\xi}(\xi-s)^{\alpha-1} f(s) d s \\
& +\frac{t^{\alpha-1}}{\Gamma(\alpha)} \int_{0}^{+\infty} f(s) d s-\frac{1}{\Gamma(\alpha)} \int_{0}^{t}(t-s)^{\alpha-1} f(s) d s+T_{1}(t) \int_{0}^{h} g_{1}(s) u(s) d s \\
= & a \sigma_{1} t^{\alpha-1}+\sigma_{1} M t^{\alpha-1} \int_{0}^{+\infty} G_{1}(\xi, s) f(s) d s+\int_{0}^{+\infty} G_{1}(t, s) f(s) d s \\
& +T_{1}(t) \int_{0}^{h} g_{1}(s) u(s) d s .
\end{aligned}
$$

Multiplying both sides of the above equality by $g_{1}(t)$ and integrating from 0 to $h$, then

$$
\begin{aligned}
\int_{0}^{h} g_{1}(t) u(t) d t= & a \sigma_{1} \int_{0}^{h} g_{1}(t) t^{\alpha-1} d t+M \sigma_{1} \int_{0}^{h} g_{1}(t) t^{\alpha-1} d t \int_{0}^{+\infty} G_{1}(\xi, s) f(s) d s \\
& +\int_{0}^{h} g_{1}(t) \int_{0}^{+\infty} G_{1}(t, s) f(s) d s d t+\int_{0}^{h} g_{1}(t) T_{1}(t) d t \int_{0}^{h} g_{1}(s) u(s) d s .
\end{aligned}
$$


Next we have

$$
\begin{aligned}
\int_{0}^{h} g_{1}(t) u(t) d t= & \frac{a \sigma_{1}}{1-\mu_{1}} \int_{0}^{h} g_{1}(t) t^{\alpha-1} d t+\frac{M \sigma_{1}}{1-\mu_{1}} \int_{0}^{h} g_{1}(t) t^{\alpha-1} d t \int_{0}^{+\infty} G_{1}(\xi, s) f(s) d s \\
& +\frac{1}{1-\mu_{1}} \int_{0}^{h} g_{1}(t) \int_{0}^{+\infty} G_{1}(t, s) f(s) d s d t .
\end{aligned}
$$

Finally, we can obtain

$$
\begin{aligned}
u(t)= & a \sigma_{1} t^{\alpha-1}+\sigma_{1} M t^{\alpha-1} \int_{0}^{+\infty} G_{1}(\xi, s) f(s) d s+\int_{0}^{+\infty} G_{1}(t, s) f(s) d s \\
& +\frac{a \sigma_{1} T_{1}(t)}{1-\mu_{1}} \int_{0}^{h} g_{1}(\tau) \tau^{\alpha-1} d \tau+\frac{T_{1}(t)}{1-\mu_{1}} \int_{0}^{h} g_{1}(\tau) \int_{0}^{+\infty} G_{1}(\tau, s) f(s) d s d \tau \\
& +\frac{M \sigma_{1} T_{1}(t)}{1-\mu_{1}} \int_{0}^{h} g_{1}(\tau) \tau^{\alpha-1} d \tau \int_{0}^{+\infty} G_{1}(\xi, s) f(s) d s \\
= & a \sigma_{1} t^{\alpha-1}+\frac{a \sigma_{1} T_{1}(t)}{1-\mu_{1}} \int_{0}^{h} g_{1}(\tau) \tau^{\alpha-1} d \tau+\int_{0}^{+\infty} H_{1}(t, s) f(s) d s \\
& +\frac{T_{1}(t)}{1-\mu_{1}} \int_{0}^{h} g_{1}(\tau) \int_{0}^{+\infty} H_{1}(\tau, s) f(s) d s d \tau \\
= & a \sigma_{1} t^{\alpha-1}+\frac{a \sigma_{1} \omega_{1} T_{1}(t)}{1-\mu_{1}}+\int_{0}^{+\infty} H(t, s) f(s) d s .
\end{aligned}
$$

This completes the proof of the lemma.

We can easily get the following result.

Lemma 2.2 The function $G_{1}(t, s)$ defined by (2.3) satisfies:

(i) $G_{1}$ is continuous and $G_{1}(t, s) \geq 0,0 \leq t, s<+\infty$;

(ii) $G_{1}(t, s)$ is increasing in $t, 0 \leq t, s<+\infty$.

Remark 2.1 For $0 \leq t, s<+\infty$, we can easily obtain

$$
\frac{G_{1}(t, s)}{1+t^{\alpha-1}} \leq \frac{1}{\Gamma(\alpha)}, \quad \frac{G_{1}(\xi, s) t^{\alpha-1}}{1+t^{\alpha-1}} \leq \frac{\xi^{\alpha-1}}{\Gamma(\alpha)}, \quad \xi>0
$$

Lemma 2.3 The function $H(t, s)$ satisfies the following inequality:

$$
\frac{H(t, s)}{1+t^{\alpha-1}} \leq \sigma_{1}+\frac{\delta_{1}\left(\sigma_{1}+\sigma_{1}^{2} M \xi^{\alpha-2}\right)}{\left(1-\mu_{1}\right) \Gamma(\alpha-1)}=\sigma_{1}\left(1+\delta_{1} l_{1}\right), \quad \forall t, s \in[0,+\infty)
$$

Proof From Remark 2.1, we have

$$
\frac{H_{1}(t, s)}{1+t^{\alpha-1}}=\frac{\sigma_{1} M t^{\alpha-1} G_{1}(\xi, s)}{1+t^{\alpha-1}}+\frac{G_{1}(t, s)}{1+t^{\alpha-1}} \leq \frac{1+\sigma_{1} M \xi^{\alpha-1}}{\Gamma(\alpha)}=\sigma_{1},
$$

thus, from (2.1), we get

$$
\frac{H(t, s)}{1+t^{\alpha-1}}=\frac{H_{1}(t, s)}{1+t^{\alpha-1}}+\frac{T_{1}(t)}{\left(1-\mu_{1}\right)\left(1+t^{\alpha-1}\right)} \int_{0}^{h} H_{1}(\tau, s) g_{1}(\tau) d \tau
$$




$$
\begin{aligned}
& \leq \sigma_{1}+\frac{\left(1+\sigma_{1} M \xi^{\alpha-2}\right) \sigma_{1}}{\left(1-\mu_{1}\right) \Gamma(\alpha-1)} \int_{0}^{h}\left(1+\tau^{\alpha-1}\right) g_{1}(\tau) d \tau \\
& =\sigma_{1}+\frac{\delta_{1}\left(\sigma_{1}+\sigma_{1}^{2} M \xi^{\alpha-2}\right)}{\left(1-\mu_{1}\right) \Gamma(\alpha-1)}=\sigma_{1}\left(1+\delta_{1} l_{1}\right) .
\end{aligned}
$$

The proof is completed.

The general solution of

$$
\begin{cases}D^{\beta} v(t)+g(t)=0, & \beta \in(2,3], t \in[0,+\infty), \\ \left.I^{3-\beta} v(t)\right|_{t=0}=0, & \left.D^{\beta-2} v(t)\right|_{t=0}=\int_{0}^{h} g_{2}(s) v(s) d s, \quad D^{\beta-1} v(+\infty)=N v(\eta)+b\end{cases}
$$

can be written by

$$
v(t)=b \sigma_{2} t^{\beta-1}+\frac{b \sigma_{2} \omega_{2} T_{2}(t)}{1-\mu_{2}}+\int_{0}^{+\infty} K(t, s) g(s) d s
$$

where

$$
\begin{aligned}
& K(t, s)=K_{1}(t, s)+\frac{T_{2}(t)}{1-\mu_{2}} \int_{0}^{h} K_{1}(\tau, s) g_{2}(\tau) d \tau, \\
& K_{1}(t, s)=\sigma_{2} N t^{\beta-1} G_{2}(\xi, s)+G_{2}(t, s),
\end{aligned}
$$

and $G_{2}(t, s)$ can be obtained from $G_{1}(t, s)$ by replacing $\alpha$ with $\beta$.

Hence, system (1.1) is equivalent to the following integral system:

$$
\left\{\begin{array}{l}
u(t)=a \sigma_{1} t^{\alpha-1}+\frac{a \sigma_{1} \omega_{1} T_{1}(t)}{1-\mu_{1}}+\int_{0}^{+\infty} H(t, s) \varphi\left(s, v(s), D^{\gamma_{1}} v(s)\right) d s \\
v(t)=b \sigma_{2} t^{\beta-1}+\frac{b \sigma_{2} \omega_{2} T_{2}(t)}{1-\mu_{2}}+\int_{0}^{+\infty} K(t, s) \psi\left(s, u(s), D^{\gamma_{2}} u(s)\right) d s
\end{array}\right.
$$

Define two spaces

$$
\begin{aligned}
& X=\left\{u \in C(J), D^{\gamma_{1}} u \in C(J) \mid \sup _{t \in J} \frac{|u(t)|}{1+t^{\alpha-1}}<+\infty, \sup _{t \in J} \frac{\left|D^{\gamma_{1}} u(t)\right|}{1+t^{\alpha-1-\gamma_{1}}}<+\infty\right\}, \\
& Y=\left\{v \in C(J), D^{\gamma_{2}} v \in C(J) \mid \sup _{t \in J} \frac{|v(t)|}{1+t^{\beta-1}}<+\infty, \sup _{t \in J} \frac{\left|D^{\gamma_{2}} v(t)\right|}{1+t^{\beta-1-\gamma_{2}}}<+\infty\right\},
\end{aligned}
$$

equipped with the norms

$$
\|u\|_{X}=\sup _{t \in J} \frac{|u(t)|}{1+t^{\alpha-1}}+\sup _{t \in J} \frac{\left|D^{\gamma_{1}} u(t)\right|}{1+t^{\alpha-1-\gamma_{1}}}, \quad\|v\|_{Y}=\sup _{t \in J} \frac{|v(t)|}{1+t^{\beta-1}}+\sup _{t \in J} \frac{\left|D^{\gamma_{2}} v(t)\right|}{1+t^{\beta-1-\gamma_{2}}}
$$

where $0<\gamma_{i}<1, i=1,2$. $C(J)$ denotes the space of all continuous functions defined on $[0,+\infty)$.

Lemma 2.4 $\left(X,\|\cdot\|_{X}\right)$ is a Banach space. 
Proof Let $\left\{u_{n}\right\}_{n=1}^{\infty}$ be a Cauchy sequence in the space $\left(X,\|\cdot\|_{X}\right)$; then $\forall \varepsilon>0, \exists N(\varepsilon)>0$ such that

$$
\left|\frac{u_{n}(t)}{1+t^{\alpha-1}}-\frac{u_{m}(t)}{1+t^{\alpha-1}}\right|+\left|\frac{D^{\gamma_{1}} u_{n}(t)}{1+t^{\alpha-1-\gamma_{1}}}-\frac{D^{\gamma_{1}} u_{m}(t)}{1+t^{\alpha-1-\gamma_{1}}}\right|<\varepsilon,
$$

for any $t \in J$ and $n, m>N(\varepsilon)$. We have $\lim _{n \rightarrow+\infty} \frac{u_{n}(t)}{1+t^{\alpha-1}}=\frac{u(t)}{1+t^{\alpha-1}}, u(t) \in C(J)$. Then, for $\frac{\Lambda_{0}}{2}=\sup _{t \in J} \frac{|u(t)|}{1+t^{\alpha-1}}>0$, there exists $N>0$ such that $\left|\frac{u_{n}(t)}{1+t^{\alpha-1}}-\frac{u(t)}{1+t^{\alpha-1}}\right|<\frac{\Lambda_{0}}{2}, n>N$. Further, set $\Lambda_{i}=\sup _{t \in J} \frac{\left|u_{i}(t)\right|}{1+t^{\alpha-1}}, i=1,2, \ldots, N$, and $\Lambda=\max \left\{\Lambda_{i}, i=0,1,2, \ldots, N\right\}$. Then $\frac{\left|u_{n}(t)\right|}{1+t^{\alpha-1}} \leq \Lambda$. Clearly, $\left\{\frac{u_{n}(t)}{1+t^{\alpha-1}}\right\}_{n=1}^{\infty}$ and $\left\{\frac{D^{\gamma_{1}} u_{n}(t)}{1+t^{\alpha-1-\gamma_{1}}}\right\}_{n=1}^{\infty}$ are Cauchy sequences in the space $C(J)$. Therefore, $\left\{\frac{D^{\gamma_{1}} u_{n}(t)}{1+t^{\alpha-1-\gamma_{1}}}\right\}_{n=1}^{\infty}$ converges uniformly to some $v \in C(J)$ and $\sup _{t \in J}|v(t)|<+\infty$. We need to prove that $v=\frac{D^{\gamma_{1}} u(t)}{1+t^{\alpha-1-\gamma_{1}}}$. For any $t \in J$, we have

$$
\begin{aligned}
& \int_{0}^{t}(t-s)^{-\gamma_{1}-1}\left(1+s^{\alpha-1}\right) \frac{u_{n}(s)}{1+s^{\alpha-1}} d s \\
& \quad \leq \Lambda \int_{0}^{t}(t-s)^{-\gamma_{1}-1}\left(1+s^{\alpha-1}\right) d s \\
& \quad=\Lambda t^{-\gamma_{1}} \int_{0}^{1}(1-\tau)^{-\gamma_{1}-1} d \tau+\Lambda t^{\alpha-1-\gamma_{1}} \int_{0}^{1} \tau^{\alpha-1}(1-\tau)^{-\gamma_{1}-1} d \tau \\
& =\Lambda t^{-\gamma_{1}} B\left(1,-\gamma_{1}\right)+\Lambda t^{\alpha-1-\gamma_{1}} B\left(\alpha,-\gamma_{1}\right) \\
& =\Lambda t^{-\gamma_{1}} \frac{\Gamma\left(-\gamma_{1}\right)}{\Gamma\left(1-\gamma_{1}\right)}+\Lambda t^{\alpha-1-\gamma_{1}} \frac{\Gamma(\alpha) \Gamma\left(-\gamma_{1}\right)}{\Gamma\left(\alpha-\gamma_{1}\right)} .
\end{aligned}
$$

Furthermore, by Lebesgue's dominated convergence theorem, and considering the uniform convergence of $\left\{\frac{D^{\gamma_{1}} u_{n}(t)}{1+t^{\alpha-1-\gamma_{1}}}\right\}_{n=1}^{\infty}$, one has

$$
\begin{aligned}
v(t) & =\lim _{n \rightarrow+\infty} \frac{D^{\gamma_{1}} u_{n}(t)}{1+t^{\alpha-1-\gamma_{1}}} \\
& =\lim _{n \rightarrow+\infty} \frac{1}{\left(1+t^{\alpha-1-\gamma_{1}}\right) \Gamma\left(1-\gamma_{1}\right)} \cdot \frac{d}{d t} \int_{0}^{t}(t-s)^{-\gamma_{1}} u_{n}(s) d s \\
& =\frac{1}{\left(1+t^{\alpha-1-\gamma_{1}}\right) \Gamma\left(1-\gamma_{1}\right)} \int_{0}^{t}(t-s)^{-\gamma_{1}-1}\left(1+s^{\alpha-1}\right) \frac{u(s)}{1+s^{\alpha-1}} d s \\
& =\frac{D^{\gamma_{1}} u(t)}{1+t^{\alpha-1-\gamma_{1}}} .
\end{aligned}
$$

Thus

$$
\lim _{n \rightarrow+\infty} \frac{u_{n}(t)}{1+t^{\alpha-1}}+\frac{D^{\gamma_{1}} u_{n}(t)}{1+t^{\alpha-1-\gamma_{1}}}=\frac{u(t)}{1+t^{\alpha-1}}+\frac{D^{\gamma_{1}} u(t)}{1+t^{\alpha-1-\gamma_{1}}} .
$$

Therefore, we conclude that $\left(X,\|\cdot\|_{X}\right)$ is a Banach space.

To prove the existence-uniqueness of solutions for system (1.1), we state the following compactness criterion.

Lemma 2.5 ([33]) Let $U \subseteq Y$ be a bounded set; then $U$ is relatively compact in $Y$ if: 
(i) for any $u \in U, \frac{u(t)}{1+t^{\alpha-1}}$ and $D^{\alpha-1} u(t)$ are equicontinuous on any compact interval of $J$;

(ii) for any $\varepsilon>0$, there exists a constant $T=T(\varepsilon)>0$ such that

$$
\left|\frac{u\left(t_{1}\right)}{1+t_{1}^{\alpha-1}}-\frac{u\left(t_{2}\right)}{1+t_{2}^{\alpha-1}}\right|<\varepsilon, \quad\left|D^{\alpha-1} u\left(t_{1}\right)-D^{\alpha-1} u\left(t_{2}\right)\right|<\varepsilon,
$$

for any $t_{1}, t_{2} \geq T$ and $u \in U$.

Remark 2.2 According to Lemmas 2.4 and 2.5, it is clear that $Z$ is relatively compact in $X$ if the following conditions hold:

(i) for any $v \in Z, \frac{v(t)}{1+t^{\alpha-1}}$ and $\frac{D^{\gamma_{1}} v(t)}{1+t^{\alpha-1-\gamma_{1}}}$ are equicontinuous on any compact interval of $J$;

(ii) for any $\varepsilon>0$, there exists a constant $L=L(\varepsilon)>0$ such that

$$
\left|\frac{v\left(t_{1}\right)}{1+t_{1}^{\alpha-1}}-\frac{v\left(t_{2}\right)}{1+t_{2}^{\alpha-1}}\right|+\left|\frac{D^{\gamma_{1}} v\left(t_{1}\right)}{1+t_{1}^{\alpha-1-\gamma_{1}}}-\frac{D^{\gamma_{1}} v\left(t_{2}\right)}{1+t_{2}^{\alpha-1-\gamma_{1}}}\right|<\varepsilon
$$

for any $t_{1}, t_{2} \geq L$ and $v \in Z$.

Lemma 2.6 (Schauder's fixed point theorem) Let $C$ be a nonempty, closed, bounded, and convex subset of a Banach space $X$. Suppose that $T: C \rightarrow C$ is a continuous and compact mapping. Then $T$ has at least one fixed point in $C$.

\section{Main results}

In our considerations, we work in the space $Q=\{(u, v) \mid u \in X, v \in Y\}$ endowed with the norm

$$
\|(u, v)\|_{Q}=\max \left\{\|u\|_{X},\|v\|_{Y}\right\}, \quad(u, v) \in Q .
$$

By Lemma 2.4, $Q$ is a Banach space. Let $T: Q \rightarrow Q$ be the operator defined as

$$
T(u, v)(t)=\left(T_{1} v(t), T_{2} u(t)\right)
$$

where

$$
\begin{aligned}
& T_{1} v(t)=a \sigma_{1} t^{\alpha-1}+\frac{a \sigma_{1} \omega_{1} T_{1}(t)}{1-\mu_{1}}+\int_{0}^{+\infty} H(t, s) \varphi\left(s, v(s), D^{\gamma_{1}} v(s)\right) d s, \\
& T_{2} u(t)=b \sigma_{2} t^{\beta-1}+\frac{b \sigma_{2} \omega_{2} T_{2}(t)}{1-\mu_{2}}+\int_{0}^{+\infty} K(t, s) \psi\left(s, u(s), D^{\gamma_{2}} u(s)\right) d s .
\end{aligned}
$$

Notice that system (1.1) has a solution if and only if the operator $T$ has a fixed point. For the forthcoming analysis, denote

$$
\begin{aligned}
& L_{1}=\sigma_{1}\left(1+\delta_{1} l_{1}\right), \quad L_{2}=\sigma_{2}\left(1+\delta_{2} l_{2}\right), \\
& \zeta_{1}=\frac{1+\sigma_{1} \Gamma(\alpha)+2 l_{1} \delta_{1}+\sigma_{1} M \xi^{\alpha-1}\left(1+2 \omega_{1} l_{1}\right)}{\Gamma\left(\alpha-\gamma_{1}\right)}, \\
& \zeta_{2}=\frac{1+\sigma_{2} \Gamma(\beta)+2 l_{2} \delta_{2}+\sigma_{2} N \eta^{\beta-1}\left(1+2 \omega_{2} l_{2}\right)}{\Gamma\left(\beta-\gamma_{2}\right)}, \\
& \theta(s)=\max \left\{1+s^{\alpha-1}, 1+s^{\alpha-1-\gamma_{1}}, 1+s^{\beta-1}, 1+s^{\beta-1-\gamma_{2}}\right\}, \quad s \in[0,+\infty) .
\end{aligned}
$$


We need the following assumptions:

$\left(H_{1}\right)$ There exist nonnegative functions $c_{i}(t), d_{i}(t) \in L^{1}(J) \cap C(J), i=1,2,3$, such that

$$
\begin{aligned}
& |\varphi(t, u, v)| \leq c_{1}(t)+c_{2}(t)|u|+c_{3}(t)|v|, \quad t \in[0,+\infty) \\
& \int_{0}^{+\infty} c_{1}(t) d t<+\infty, \quad \int_{0}^{+\infty}\left(c_{2}(t)+c_{3}(t)\right) \theta(t) d t<\max \left\{\frac{1}{2 L_{1}}, \frac{1}{2 \zeta_{1}}\right\}
\end{aligned}
$$

and

$$
\begin{aligned}
& |\psi(t, u, v)| \leq d_{1}(t)+d_{2}(t)|u|+d_{3}(t)|v|, \quad t \in[0,+\infty) \\
& \int_{0}^{+\infty} d_{1}(t) d t<+\infty, \quad \int_{0}^{+\infty}\left(d_{2}(t)+d_{3}(t)\right) \theta(t) d t<\max \left\{\frac{1}{2 L_{2}}, \frac{1}{2 \zeta_{2}}\right\} .
\end{aligned}
$$

$\left(H_{2}\right)$ For any $u, v, x, y \in \mathbb{R}$, there exist $\lambda_{i}(t) \in L^{1}(J) \cap C(J)$ with $\lambda_{i}(t)>0, i=1,2$, such that

$$
\begin{aligned}
& |\varphi(t, u, v)-\varphi(t, x, y)| \leq \lambda_{1}(t)(|u-x|+|v-y|), \quad t \in[0,+\infty), \\
& |\psi(t, u, v)-\psi(t, x, y)| \leq \lambda_{2}(t)(|u-x|+|v-y|), \quad t \in[0,+\infty) .
\end{aligned}
$$

This section is devoted to some existence and uniqueness results of system (1.1). In order to do this, define

$$
B_{R}=\left\{(u, v) \in Q \mid\|(u, v)\|_{Q} \leq R\right\}
$$

where

$$
\begin{aligned}
R> & \left\{\frac{a \sigma_{1}\left(1+\omega_{1} l_{1}\right)+L_{1} \int_{0}^{+\infty} c_{1}(s) d s}{\frac{1}{2}-L_{1} \int_{0}^{+\infty}\left(c_{2}(s)+c_{3}(s)\right) \theta(s) d s}, \frac{\frac{2 a \sigma_{1} \Gamma(\alpha-1)}{\Gamma\left(\alpha-\gamma_{1}\right)}\left(1+\omega_{1} l_{1}\right)+\zeta_{1} \int_{0}^{+\infty} c_{1}(s) d s}{\frac{1}{2}-\zeta_{1} \int_{0}^{+\infty}\left(c_{2}(s)+c_{3}(s)\right) \theta(s) d s},\right. \\
& \left.\frac{b \sigma_{2}\left(1+\omega_{2} l_{2}\right)+L_{2} \int_{0}^{+\infty} d_{1}(s) d s}{\frac{1}{2}-L_{2} \int_{0}^{+\infty}\left(d_{2}(s)+d_{3}(s)\right) \theta(s) d s}, \frac{\frac{2 b \sigma_{2} \Gamma(\beta-1)}{\Gamma\left(\beta-\gamma_{2}\right)}\left(1+\omega_{2} l_{2}\right)+\zeta_{2} \int_{0}^{+\infty} d_{1}(s) d s}{\frac{1}{2}-\zeta_{2} \int_{0}^{+\infty}\left(d_{2}(s)+d_{3}(s)\right) \theta(s) d s}\right\}
\end{aligned}
$$

We observe that $B_{R}$ is a bounded closed ball in the Banach space $Q$.

Lemma 3.1 If $\left(H_{1}\right)$ is satisfied, then $T: B_{R} \rightarrow B_{R}$.

Proof First, for any $(u, v) \in B_{R}$, we know that

$$
\left\|T_{1} v\right\|_{X}=\sup _{t \in J} \frac{\left|T_{1} v(t)\right|}{1+t^{\alpha-1}}+\sup _{t \in J} \frac{\left|D^{\gamma_{1}} T_{1} v(t)\right|}{1+t^{\alpha-1-\gamma_{1}}}
$$

and from condition $\left(H_{1}\right)$, we have

$$
\begin{aligned}
\frac{\left|T_{1} v(t)\right|}{1+t^{\alpha-1}} & =\left|\frac{a \sigma_{1} t^{\alpha-1}}{1+t^{\alpha-1}}+\frac{T_{1}(t)}{1+t^{\alpha-1}} \cdot \frac{a \sigma_{1} \omega_{1}}{1-\mu_{1}}+\int_{0}^{+\infty} \frac{H(t, s)}{1+t^{\alpha-1}} \varphi\left(s, v(s), D^{\gamma_{1}} v(s)\right) d s\right| \\
& \leq a \sigma_{1}+\frac{a \sigma_{1} \omega_{1}\left(1+\sigma_{1} M \xi^{\alpha-2}\right)}{\left(1-\mu_{1}\right) \Gamma(\alpha-1)}+\int_{0}^{+\infty} \sigma_{1}\left(1+\delta_{1} l_{1}\right)\left|\varphi\left(s, v(s), D^{\gamma_{1}} v(s)\right)\right| d s \\
& \leq a \sigma_{1}\left(1+\omega_{1} l_{1}\right)+L_{1} \int_{0}^{+\infty}\left(c_{1}(s)+c_{2}(s)|v(s)|+c_{3}(s)\left|D^{\gamma_{1}} v(s)\right|\right) d s
\end{aligned}
$$




$$
\begin{aligned}
& \leq a \sigma_{1}\left(1+\omega_{1} l_{1}\right)+L_{1} \int_{0}^{+\infty} c_{1}(s) d s+L_{1} \int_{0}^{+\infty}\left(c_{2}(s)+c_{3}(s)\right) \theta(s) d s\|(v, v)\|_{Q} \\
& <\frac{a \sigma_{1}\left(1+\omega_{1} l_{1}\right)+L_{1} \int_{0}^{+\infty} c_{1}(s) d s}{1-2 L_{1} \int_{0}^{+\infty}\left(c_{2}(s)+c_{3}(s)\right) \theta(s) d s}<\frac{R}{2}
\end{aligned}
$$

In view of Lemma 2.1, one has

$$
\begin{aligned}
D^{\gamma_{1}} u(t)= & D^{\gamma_{1}}\left(\sigma_{1} t^{\alpha-1}\left(a+\int_{0}^{+\infty} f(s) d s+\frac{M \xi^{\alpha-2}}{\Gamma(\alpha-1)} \int_{0}^{h} g_{1}(s) u(s) d s-M I^{\alpha} f(\xi)\right)\right. \\
& \left.+\frac{t^{\alpha-2}}{\Gamma(\alpha-1)} \int_{0}^{h} g_{1}(s) u(s) d s-I^{\alpha} f(t)\right) \\
= & \left(a+\int_{0}^{+\infty} f(s) d s+\frac{M \xi^{\alpha-2}}{\Gamma(\alpha-1)} \int_{0}^{h} g_{1}(s) u(s) d s-M I^{\alpha} f(\xi)\right) \cdot \frac{\sigma_{1} t^{\alpha-1-\gamma_{1}} \Gamma(\alpha)}{\Gamma\left(\alpha-\gamma_{1}\right)} \\
& +\frac{t^{\alpha-2-\gamma_{1}}}{\Gamma\left(\alpha-1-\gamma_{1}\right)} \int_{0}^{h} g_{1}(s) u(s) d s-I^{\alpha-\gamma_{1}} f(t)
\end{aligned}
$$

and thus, we can easily show that

$$
\begin{aligned}
\left|\int_{0}^{h} g_{1}(t) T_{1} v(t) d t\right|= & \frac{a \sigma_{1}}{1-\mu_{1}} \int_{0}^{h} g_{1}(t) t^{\alpha-1} d t \\
& +\frac{M \sigma_{1}}{1-\mu_{1}} \int_{0}^{h} g_{1}(t) t^{\alpha-1} d t \int_{0}^{+\infty} G_{1}(\xi, s) \varphi\left(s, v(s), D^{\gamma_{1}} v(s)\right) d s \\
& +\frac{1}{1-\mu_{1}} \int_{0}^{h} g_{1}(t) \int_{0}^{+\infty} G_{1}(t, s) \varphi\left(s, v(s), D^{\gamma_{1}} v(s)\right) d s d t \mid \\
\leq & \frac{a \sigma_{1} \omega_{1}}{1-\mu_{1}}+\frac{\sigma_{1} \omega_{1} M \xi^{\alpha-1}}{\left(1-\mu_{1}\right) \Gamma(\alpha)} \int_{0}^{+\infty}\left|\varphi\left(s, v(s), D^{\gamma_{1}} v(s)\right)\right| d s \\
& +\frac{1}{\left(1-\mu_{1}\right) \Gamma(\alpha)} \int_{0}^{h} g_{1}(t)\left(1+t^{\alpha-1}\right) d t \int_{0}^{+\infty}\left|\varphi\left(s, v(s), D^{\gamma_{1}} v(s)\right)\right| d s \\
\leq & \frac{a \sigma_{1} \omega_{1}}{1-\mu_{1}}+\frac{\sigma_{1} \omega_{1} M \xi^{\alpha-1}+\delta_{1}}{\left(1-\mu_{1}\right) \Gamma(\alpha)} \int_{0}^{+\infty} c_{1}(s) d s \\
& +\frac{\sigma_{1} \omega_{1} M \xi^{\alpha-1}+\delta_{1}}{\left(1-\mu_{1}\right) \Gamma(\alpha)} \int_{0}^{+\infty}\left(c_{2}(s)+c_{3}(s)\right) \theta(s) d s\|(v, v)\|_{Q} .
\end{aligned}
$$

Further,

$$
\begin{aligned}
\frac{\left|D^{\gamma_{1}} T_{1} v(t)\right|}{1+t^{\alpha-1-\gamma_{1}}}= & \mid \frac{\sigma_{1} t^{\alpha-1-\gamma_{1}} \Gamma(\alpha)}{\left(1+t^{\alpha-1-\gamma_{1}}\right) \Gamma\left(\alpha-\gamma_{1}\right)}\left(a+\int_{0}^{+\infty} \varphi\left(s, v(s), D^{\gamma_{1}} v(s)\right) d s\right. \\
& +\frac{M \xi^{\alpha-2}}{\Gamma(\alpha-1)} \int_{0}^{h} g_{1}(s) T_{1} v(s) d s \\
& \left.-\frac{M}{\Gamma(\alpha)} \int_{0}^{\xi}(\xi-s)^{\alpha-1} \varphi\left(s, v(s), D^{\gamma_{1}} v(s)\right) d s\right) \\
& +\frac{t^{\alpha-2-\gamma_{1}}}{\left(1+t^{\alpha-1-\gamma_{1}}\right)} \int_{0}^{h} g_{1}(s) \frac{T_{1} v(s)}{\Gamma\left(\alpha-1-\gamma_{1}\right)} d s \\
& -\frac{1}{\Gamma\left(\alpha-\gamma_{1}\right)} \int_{0}^{t} \frac{(t-s)^{\alpha-1-\gamma_{1}}}{1+t^{\alpha-1-\gamma_{1}}} \varphi\left(s, \nu(s), D^{\gamma_{1}} v(s)\right) d s \mid
\end{aligned}
$$




$$
\begin{aligned}
& \leq \frac{a \sigma_{1} \Gamma(\alpha)}{\Gamma\left(\alpha-\gamma_{1}\right)}+\frac{\sigma_{1} \Gamma(\alpha)}{\Gamma\left(\alpha-\gamma_{1}\right)} \int_{0}^{+\infty}\left|\varphi\left(s, v(s), D^{\gamma_{1}} v(s)\right)\right| d s \\
& +\frac{\sigma_{1} M \xi^{\alpha-1}}{\Gamma\left(\alpha-\gamma_{1}\right)} \int_{0}^{\xi}\left|\varphi\left(s, v(s), D^{\gamma_{1}} v(s)\right)\right| d s \\
& +\frac{\sigma_{1} M \xi^{\alpha-2}(\alpha-1)+\alpha-1-\gamma_{1}}{\Gamma\left(\alpha-\gamma_{1}\right)}\left|\int_{0}^{h} g_{1}(s) T_{1} v(s) d s\right| \\
& +\frac{1}{\Gamma\left(\alpha-\gamma_{1}\right)} \int_{0}^{t}\left(1-\frac{s}{t}\right)^{\alpha-1-\gamma_{1}}\left|\varphi\left(s, v(s), D^{\gamma_{1}} v(s)\right)\right| d s \\
& \leq \frac{1+\sigma_{1} \Gamma(\alpha)+2 l_{1} \delta_{1}+\sigma_{1} M \xi^{\alpha-1}\left(1+2 \omega_{1} l_{1}\right)}{\Gamma\left(\alpha-\gamma_{1}\right)} \int_{0}^{+\infty} c_{1}(s) d s \\
& +\frac{1+\sigma_{1} \Gamma(\alpha)+2 l_{1} \delta_{1}+\sigma_{1} M \xi^{\alpha-1}\left(1+2 \omega_{1} l_{1}\right)}{\Gamma\left(\alpha-\gamma_{1}\right)} \\
& \times \int_{0}^{+\infty}\left(c_{2}(s)+c_{3}(s)\right) \theta(s) d s\|(v, v)\|_{Q} \\
& +a \sigma_{1}\left(\frac{\Gamma(\alpha)}{\Gamma\left(\alpha-\gamma_{1}\right)}+\frac{2 \omega_{1} l_{1} \Gamma(\alpha-1)}{\Gamma\left(\alpha-\gamma_{1}\right)}\right) \\
& <\frac{\frac{2 a \sigma_{1} \Gamma(\alpha-1)}{\Gamma\left(\alpha-\gamma_{1}\right)}\left(1+\omega_{1} l_{1}\right)+\zeta_{1} \int_{0}^{+\infty} c_{1}(s) d s}{1-2 \zeta_{1} \int_{0}^{+\infty}\left(c_{2}(s)+c_{3}(s)\right) \theta(s) d s}<\frac{R}{2},
\end{aligned}
$$

which implies that

$$
\left\|T_{1} v\right\|_{X}=\sup _{t \in J} \frac{\left|T_{1} v(t)\right|}{1+t^{\alpha-1}}+\sup _{t \in J} \frac{\left|D^{\gamma_{1}} T_{1} v(t)\right|}{1+t^{\alpha-1-\gamma_{1}}}<\frac{R}{2}+\frac{R}{2}=R .
$$

Similarly, we can obtain

$$
\begin{aligned}
\left\|T_{2} u\right\|_{Y} & =\sup _{t \in J} \frac{\left|T_{2} u(t)\right|}{1+t^{\beta-1}}+\sup _{t \in J} \frac{\left|D^{\gamma_{2}} T_{2} u(t)\right|}{1+t^{\beta-1-\gamma_{2}}} \\
& \leq \frac{b \sigma_{2}\left(1+\omega_{2} l_{2}\right)+L_{2} \int_{0}^{+\infty} d_{1}(s) d s}{\frac{1}{2}-L_{2} \int_{0}^{+\infty}\left(d_{2}(s)+d_{3}(s)\right) \theta(s) d s}+\frac{\frac{2 b \sigma_{2} \Gamma(\beta-1)}{\Gamma\left(\beta-\gamma_{2}\right)}\left(1+\omega_{2} l_{2}\right)+\zeta_{2} \int_{0}^{+\infty} d_{1}(s) d s}{\frac{1}{2}-\zeta_{2} \int_{0}^{+\infty}\left(d_{2}(s)+d_{3}(s)\right) \theta(s) d s} \\
& <\frac{R}{2}+\frac{R}{2}=R .
\end{aligned}
$$

It shows that $\|T(u, v)\|_{Q} \leq R$, and $T_{1}, T_{2}$ are continuous on $J$. Thus $T: B_{R} \rightarrow B_{R}$ is well defined.

Theorem 3.1 If $\left(H_{1}\right)$ holds, then system (1.1) has at least one solution.

Proof First, the operator $T: B_{R} \rightarrow B_{R}$ is continuous owing to the continuity of $\varphi$ and $\psi$. We are going to show that $T$ is a completely continuous operator. By Lemma 3.1, $T$ is bounded. We need to show that $T$ is relatively compact by means of Remark 2.2. This part consists of two steps as follows.

Step 1 We show that $T$ is equicontinuous on any compact interval of $J$. 
Let $\omega$ be a bounded subset of $B_{R}, J_{1} \subseteq[0,+\infty)$ be a compact interval. Then, for any $t_{1}, t_{2} \in J_{1}$ with $t_{1}<t_{2}, v \in \omega$, we have

$$
\begin{aligned}
& \left|\frac{T_{1} v\left(t_{2}\right)}{1+t_{2}^{\alpha-1}}-\frac{T_{1} v\left(t_{1}\right)}{1+t_{1}^{\alpha-1}}\right| \\
& =\mid \frac{a \sigma_{1} t_{2}^{\alpha-1}}{1+t_{2}^{\alpha-1}}-\frac{a \sigma_{1} t_{1}^{\alpha-1}}{1+t_{1}^{\alpha-1}}+\int_{0}^{+\infty}\left(\frac{H\left(t_{2}, s\right)}{1+t_{2}^{\alpha-1}}-\frac{H\left(t_{1}, s\right)}{1+t_{1}^{\alpha-1}}\right) \varphi\left(s, v(s), D^{\gamma_{1}} v(s)\right) d s \\
& +\left(\frac{T_{1}\left(t_{2}\right)}{\left(1-\mu_{1}\right)\left(1+t_{2}^{\alpha-1}\right)}-\frac{T_{1}\left(t_{1}\right)}{\left(1-\mu_{1}\right)\left(1+t_{1}^{\alpha-1}\right)}\right) \cdot a \sigma_{1} \omega_{1} \mid \\
& \leq \frac{\left|t_{2}^{\alpha-1}-t_{1}^{\alpha-1}\right| a \sigma_{1}}{\left(1+t_{2}^{\alpha-1}\right)\left(1+t_{1}^{\alpha-1}\right)}+\left|\frac{\sigma_{1} M t_{2}^{\alpha-1}}{1+t_{2}^{\alpha-1}}-\frac{\sigma_{1} M t_{1}^{\alpha-1}}{1+t_{1}^{\alpha-1}}\right| \int_{0}^{+\infty} G_{1}(\xi, s)\left|\varphi\left(s, \nu(s), D^{\gamma_{1}} v(s)\right)\right| d s \\
& +\left|\frac{T_{1}\left(t_{2}\right)}{\left(1-\mu_{1}\right)\left(1+t_{2}^{\alpha-1}\right)}-\frac{T_{1}\left(t_{1}\right)}{\left(1-\mu_{1}\right)\left(1+t_{1}^{\alpha-1}\right)}\right| \\
& \times \int_{0}^{+\infty} \int_{0}^{h} H_{1}(\tau, s) g_{1}(\tau)\left|\varphi\left(s, \nu(s), D^{\gamma_{1}} v(s)\right)\right| d \tau d s \\
& +\int_{0}^{+\infty} \frac{G_{1}\left(t_{2}, s\right)-G_{1}\left(t_{1}, s\right)}{1+t_{2}^{\alpha-1}}\left|\varphi\left(s, v(s), D^{\gamma_{1}} v(s)\right)\right| d s \\
& +\int_{0}^{+\infty}\left|\frac{G_{1}\left(t_{1}, s\right)}{1+t_{2}^{\alpha-1}}-\frac{G_{1}\left(t_{1}, s\right)}{1+t_{1}^{\alpha-1}}\right|\left|\varphi\left(s, v(s), D^{\gamma_{1}} v(s)\right)\right| d s \\
& \leq \frac{\sigma_{1} M \xi^{\alpha-2}\left|t_{2}^{\alpha-1}-t_{1}^{\alpha-1}\right|+\left|t_{2}^{\alpha-2}-t_{1}^{\alpha-2}\right|+\left(t_{1} t_{2}\right)^{\alpha-2}\left|t_{1}-t_{2}\right|}{\left(1+t_{2}^{\alpha-1}\right)\left(1+t_{1}^{\alpha-1}\right)\left(1-\mu_{1}\right) \Gamma(\alpha-1)} \\
& \times\left(a \sigma_{1} \omega_{1}+\sigma_{1} \delta_{1} \int_{0}^{+\infty}\left|\varphi\left(s, v(s), D^{\gamma_{1}} v(s)\right)\right| d s\right) \\
& +\frac{\left|t_{2}^{\alpha-1}-t_{1}^{\alpha-1}\right| a \sigma_{1}}{\left(1+t_{2}^{\alpha-1}\right)\left(1+t_{1}^{\alpha-1}\right)}+\frac{\left|t_{2}^{\alpha-1}-t_{1}^{\alpha-1}\right| \sigma_{1} M \xi^{\alpha-1}}{\left(1+t_{2}^{\alpha-1}\right)\left(1+t_{1}^{\alpha-1}\right) \Gamma(\alpha)} \int_{0}^{+\infty}\left|\varphi\left(s, v(s), D^{\gamma_{1}} v(s)\right)\right| d s \\
& +\int_{0}^{t_{1}}\left|\frac{\left(t_{2}^{\alpha-1}-t_{1}^{\alpha-1}\right)+\left(t_{1}-s\right)^{\alpha-1}-\left(t_{2}-s\right)^{\alpha-1}}{\Gamma(\alpha)\left(1+t_{2}^{\alpha-1}\right)}\right|\left|\varphi\left(s, v(s), D^{\gamma_{1}} v(s)\right)\right| d s \\
& +\int_{t_{1}}^{t_{2}}\left|\frac{\left(t_{2}^{\alpha-1}-t_{1}^{\alpha-1}\right)-\left(t_{2}-s\right)^{\alpha-1}}{\Gamma(\alpha)\left(1+t_{2}^{\alpha-1}\right)}\right| \varphi\left(s, v(s), D^{\gamma_{1}} v(s)\right) \mid d s \\
& +\int_{t_{2}}^{+\infty}\left|\frac{t_{2}^{\alpha-1}-t_{1}^{\alpha-1}}{\Gamma(\alpha)\left(1+t_{2}^{\alpha-1}\right)}\right|\left|\varphi\left(s, v(s), D^{\gamma_{1}} v(s)\right)\right| d s \\
& +\int_{0}^{+\infty}\left|\frac{t_{1}^{\alpha-1}-t_{2}^{\alpha-1}}{\Gamma(\alpha)\left(1+t_{2}^{\alpha-1}\right)}\right| \varphi\left(s, v(s), D^{\gamma_{1}} v(s)\right) \mid d s .
\end{aligned}
$$

Then we have $\left|\frac{T_{1} v\left(t_{2}\right)}{1+t_{2}^{\alpha-1}}-\frac{T_{1} v\left(t_{1}\right)}{1+t_{1}^{\alpha-1}}\right| \rightarrow 0$ as $t_{1} \rightarrow t_{2}$. Further, we know that

$$
\begin{aligned}
& \left|\frac{D^{\gamma_{1}} T_{1} v\left(t_{2}\right)}{1+t_{2}^{\alpha-1-\gamma_{1}}}-\frac{D^{\gamma_{1}} T_{1} v\left(t_{1}\right)}{1+t_{1}^{\alpha-1-\gamma_{1}}}\right| \\
& =\mid \frac{t_{1}^{\alpha-1-\gamma_{1}}-t_{2}^{\alpha-1-\gamma_{1}}}{\left(1+t_{1}^{\alpha-1-\gamma_{1}}\right)\left(1+t_{2}^{\alpha-1-\gamma_{1}}\right)}\left(\frac{a \sigma_{1} \Gamma(\alpha)}{\Gamma\left(\alpha-\gamma_{1}\right)}+\frac{\sigma_{1} \Gamma(\alpha)}{\Gamma\left(\alpha-\gamma_{1}\right)} \int_{0}^{+\infty} \varphi\left(s, v(s), D^{\gamma_{1}} v(s)\right) d s\right. \\
& -\frac{\sigma_{1} M}{\Gamma\left(\alpha-\gamma_{1}\right)} \int_{0}^{\xi}(\xi-s)^{\alpha-1} \varphi\left(s, \nu(s), D^{\gamma_{1}} v(s)\right) d s
\end{aligned}
$$




$$
\begin{aligned}
& \left.+\frac{\sigma_{1} M \xi^{\alpha-2}(\alpha-1)}{\Gamma\left(\alpha-\gamma_{1}\right)} \int_{0}^{h} g_{1}(s) T_{1} v(s) d s\right) \\
& +\left(\frac{t_{2}^{\alpha-1-\gamma_{1}}}{1+t_{2}^{\alpha-1-\gamma_{1}}}-\frac{t_{1}^{\alpha-1-\gamma_{1}}}{1+t_{1}^{\alpha-1-\gamma_{1}}}\right) \int_{0}^{h} g_{1}(s) \frac{T_{1} v(s)}{\Gamma\left(\alpha-1-\gamma_{1}\right)} d s \\
& +\frac{1}{\Gamma\left(\alpha-\gamma_{1}\right)} \int_{0}^{t_{1}} \frac{\left(t_{1}-s\right)^{\alpha-1-\gamma_{1}}}{1+t_{1}^{\alpha-1-\gamma_{1}}} \varphi\left(s, v(s), D^{\gamma_{1}} v(s)\right) d s \\
& -\frac{1}{\Gamma\left(\alpha-\gamma_{1}\right)} \int_{0}^{t_{2}} \frac{\left(t_{2}-s\right)^{\alpha-1-\gamma_{1}}}{1+t_{2}^{\alpha-1-\gamma_{1}}} \varphi\left(s, v(s), D^{\gamma_{1}} v(s)\right) d s \mid \\
& \leq \frac{\left|t_{2}^{\alpha-1-\gamma_{1}}-t_{1}^{\alpha-1-\gamma_{1}}\right|}{\left(1+t_{1}^{\alpha-1-\gamma_{1}}\right)\left(1+t_{2}^{\alpha-1-\gamma_{1}}\right)}\left(\frac{a \sigma_{1} \Gamma(\alpha)}{\Gamma\left(\alpha-\gamma_{1}\right)}+\frac{\sigma_{1} \Gamma(\alpha)}{\Gamma\left(\alpha-\gamma_{1}\right)} \int_{0}^{+\infty}\left|\varphi\left(s, v(s), D^{\gamma_{1}} v(s)\right)\right| d s\right. \\
& \left.+\frac{\sigma_{1} M \xi^{\alpha-1}}{\Gamma\left(\alpha-\gamma_{1}\right)} \int_{0}^{\xi}\left|\varphi\left(s, v(s), D^{\gamma_{1}} v(s)\right)\right| d s+\frac{2 \sigma_{1} M \xi^{\alpha-2}}{\Gamma\left(\alpha-\gamma_{1}\right)} \int_{0}^{h} g_{1}(s) T_{1} v(s) d s\right) \\
& +\frac{\left|t_{2}^{\alpha-1-\gamma_{1}}-t_{1}^{\alpha-1-\gamma_{1}}\right|}{\left(1+t_{1}^{\alpha-1-\gamma_{1}}\right)\left(1+t_{2}^{\alpha-1-\gamma_{1}}\right) \Gamma\left(\alpha-1-\gamma_{1}\right)} \int_{0}^{h} g_{1}(s) T_{1} v(s) d s \\
& +\frac{1}{\Gamma\left(\alpha-\gamma_{1}\right)} \int_{0}^{t_{1}}\left|\frac{\left(t_{1}-s\right)^{\alpha-1-\gamma_{1}}}{1+t_{1}^{\alpha-1-\gamma_{1}}}-\frac{\left(t_{2}-s\right)^{\alpha-1-\gamma_{1}}}{1+t_{2}^{\alpha-1-\gamma_{1}}}\right|\left|\varphi\left(s, v(s), D^{\gamma_{1}} v(s)\right)\right| d s \\
& +\frac{1}{\Gamma\left(\alpha-\gamma_{1}\right)} \int_{t_{1}}^{t_{2}} \frac{\left(t_{2}-s\right)^{\alpha-1-\gamma_{1}}}{1+t_{2}^{\alpha-1-\gamma_{1}}}\left|\varphi\left(s, v(s), D^{\gamma_{1}} v(s)\right)\right| d s,
\end{aligned}
$$

so $\left|\frac{D^{\gamma_{1}} T_{1} v\left(t_{2}\right)}{1+t_{2}^{\alpha-1-\gamma_{1}}}-\frac{D^{\gamma_{1}} T_{1} v\left(t_{1}\right)}{1+t_{1}^{\alpha-1-\gamma_{1}}}\right| \rightarrow 0$ as $t_{1} \rightarrow t_{2}$. Moreover, notice that $\varphi\left(t, v(t), D^{\gamma_{1}} v(t)\right)$ is bounded on $J_{1}$. For any $v \in \omega, \frac{T_{1} v(t)}{1+t^{\alpha-1}}$ and $\frac{D^{\gamma_{1}} T_{1} v(t)}{1+t^{\alpha-1-\gamma_{1}}}$ are equicontinuous on $J_{1}$, that is, $T_{1}$ is equicontinuous. Similarly, we know that $T_{2}$ is also equicontinuous. Thus $T$ is equicontinuous on $J_{1}$.

Step 2 We show that $T$ is equiconvergent at $\infty$.

Since $\lim _{t \rightarrow+\infty} \frac{t^{\lambda-1}}{1+t^{\lambda-1}}=1$, for any $\varepsilon>0$, there exists a constant $\mu_{1}>0$, for each $t>\mu_{1}$, one has $\left|\frac{t^{\lambda-1}}{1+t^{\lambda-1}}-1\right|<\frac{\varepsilon}{2}$. Thus, for each $t_{1}, t_{2}>\mu_{1}$, we have

$$
\left|\frac{t_{2}^{\lambda-1}}{1+t_{2}^{\lambda-1}}-\frac{t_{1}^{\lambda-1}}{1+t_{1}^{\lambda-1}}\right| \leq\left|\frac{t_{2}^{\lambda-1}}{1+t_{2}^{\lambda-1}}-1\right|+\left|\frac{t_{1}^{\lambda-1}}{1+t_{1}^{\lambda-1}}-1\right|<\frac{\varepsilon}{2}+\frac{\varepsilon}{2}=\varepsilon
$$

Further, there exists $\zeta \geq s$ such that $\lim _{t \rightarrow+\infty} \frac{(t-\varsigma)^{\lambda-1}}{1+t^{\lambda-1}}=1$. Then, for any $\varepsilon>0$, there exists $\mu_{2}>\varsigma>0$ such that, for each $t_{1}, t_{2}>\mu_{2}$, we have

$$
\begin{aligned}
\left|\frac{\left(t_{2}-s\right)^{\lambda-1}}{1+t_{2}^{\lambda-1}}-\frac{\left(t_{1}-s\right)^{\lambda-1}}{1+t_{1}^{\lambda-1}}\right| & \leq\left|\frac{\left(t_{2}-\varsigma\right)^{\lambda-1}}{1+t_{2}^{\lambda-1}}-1\right|+\left|\frac{\left(t_{1}-\varsigma\right)^{\lambda-1}}{1+t_{1}^{\lambda-1}}-1\right| \\
& <\frac{\varepsilon}{2}+\frac{\varepsilon}{2}=\varepsilon .
\end{aligned}
$$

Therefore, for any $\varepsilon>0$, choose $\mu \geq \max \left\{\mu_{1}, \mu_{2}\right\}$; then, for each $t_{1}, t_{2}>\mu$, one has

$$
\begin{aligned}
& \left|\frac{T_{1} v\left(t_{2}\right)}{1+t_{2}^{\alpha-1}}-\frac{T_{1} v\left(t_{1}\right)}{1+t_{1}^{\alpha-1}}\right| \\
& \quad \leq\left|\frac{t_{2}^{\alpha-1}}{1+t_{2}^{\alpha-1}}-\frac{t_{1}^{\alpha-1}}{1+t_{1}^{\alpha-1}}\right| a \sigma_{1}+\left(\frac{\sigma_{1} M \xi^{\alpha-2}}{\Gamma(\alpha-1)\left(1-\mu_{1}\right)}\left|\frac{t_{2}^{\alpha-1}}{1+t_{2}^{\alpha-1}}-\frac{t_{1}^{\alpha-1}}{1+t_{1}^{\alpha-1}}\right|\right.
\end{aligned}
$$




$$
\begin{aligned}
& \left.+\frac{1}{\Gamma(\alpha-1)\left(1-\mu_{1}\right)}\left|\frac{t_{2}^{\alpha-2}}{1+t_{2}^{\alpha-1}}-\frac{t_{1}^{\alpha-2}}{1+t_{1}^{\alpha-1}}\right|\right) \\
& \times\left(a \sigma_{1} \omega_{1}+\int_{0}^{+\infty} \int_{0}^{h} H_{1}(\tau, s) g_{1}(\tau)\left|\varphi\left(s, v(s), D^{\gamma_{1}} v(s)\right)\right| d \tau d s\right) \\
& +\left|\frac{t_{2}^{\alpha-1}}{1+t_{2}^{\alpha-1}}-\frac{t_{1}^{\alpha-1}}{1+t_{1}^{\alpha-1}}\right| \cdot \frac{\sigma_{1} M \xi^{\alpha-1}}{\Gamma(\alpha)} \int_{0}^{+\infty}\left|\varphi\left(s, v(s), D^{\gamma_{1}} v(s)\right)\right| d s \\
& +\frac{1}{\Gamma(\alpha)} \int_{0}^{t_{1}}\left(\left|\frac{t_{2}^{\alpha-1}}{1+t_{2}^{\alpha-1}}-\frac{t_{1}^{\alpha-1}}{1+t_{1}^{\alpha-1}}\right|+\left|\frac{\left(t_{1}-s\right)^{\alpha-1}}{1+t_{1}^{\alpha-1}}-\frac{\left(t_{2}-s\right)^{\alpha-1}}{1+t_{2}^{\alpha-1}}\right|\right) \\
& \times\left|\varphi\left(s, v(s), D^{\gamma_{1}} v(s)\right)\right| d s \\
& +\frac{1}{\Gamma(\alpha)} \int_{t_{1}}^{+\infty}\left|\frac{t_{2}^{\alpha-1}}{1+t_{2}^{\alpha-1}}-\frac{t_{1}^{\alpha-1}}{1+t_{1}^{\alpha-1}}\right| \varphi\left(s, v(s), D^{\gamma_{1}} v(s)\right) \mid d s \\
& \leq \varepsilon a \sigma_{1}+\frac{\varepsilon\left(1+\sigma_{1} M \xi^{\alpha-2}\right)}{\Gamma(\alpha-1)\left(1-\mu_{1}\right)}\left(a \sigma_{1} \omega_{1}+\sigma_{1} \delta_{1} \int_{0}^{+\infty}\left|\varphi\left(s, v(s), D^{\gamma_{1}} v(s)\right)\right| d s\right) \\
& +\frac{\varepsilon \sigma_{1} M \xi^{\alpha-1}}{\Gamma(\alpha)} \int_{0}^{+\infty}\left|\varphi\left(s, v(s), D^{\gamma_{1}} v(s)\right)\right| d s+\frac{2 \varepsilon}{\Gamma(\alpha)} \int_{0}^{t_{1}}\left|\varphi\left(s, v(s), D^{\gamma_{1}} v(s)\right)\right| d s \\
& +\frac{\varepsilon}{\Gamma(\alpha)} \int_{t_{1}}^{+\infty}\left|\varphi\left(s, v(s), D^{\gamma_{1}} v(s)\right)\right| d s \\
& \leq \varepsilon a \sigma_{1}\left(1+\omega_{1} l_{1}\right)+\varepsilon\left(L_{1}+\frac{2}{\Gamma(\alpha)}\right) \int_{0}^{+\infty}\left(c_{1}(s)+c_{2}(s)|v(s)|+c_{3}(s)\left|D^{\gamma_{1}} v(s)\right|\right) d s .
\end{aligned}
$$

In addition, we can obtain

$$
\begin{aligned}
& \left|\frac{D^{\gamma_{1}} T_{1} v\left(t_{2}\right)}{1+t_{2}^{\alpha-1-\gamma_{1}}}-\frac{D^{\gamma_{1}} T_{1} v\left(t_{1}\right)}{1+t_{1}^{\alpha-1-\gamma_{1}}}\right| \\
& \leq\left|\frac{t_{2}^{\alpha-1-\gamma_{1}}}{1+t_{2}^{\alpha-1-\gamma_{1}}}-\frac{t_{1}^{\alpha-1-\gamma_{1}}}{1+t_{1}^{\alpha-1-\gamma_{1}}}\right|\left(\frac{a \sigma_{1} \Gamma(\alpha)}{\Gamma\left(\alpha-\gamma_{1}\right)}+\frac{\sigma_{1} \Gamma(\alpha)}{\Gamma\left(\alpha-\gamma_{1}\right)} \int_{0}^{+\infty}\left|\varphi\left(s, v(s), D^{\gamma_{1}} v(s)\right)\right| d s\right. \\
& +\frac{M \sigma_{1}}{\Gamma\left(\alpha-\gamma_{1}\right)} \int_{0}^{\xi}(\xi-s)^{\alpha-1}\left|\varphi\left(s, v(s), D^{\gamma_{1}} v(s)\right)\right| d s \\
& \left.+\frac{\sigma_{1} M \xi^{\alpha-2}(\alpha-1)+\alpha-1-\gamma_{1}}{\Gamma\left(\alpha-\gamma_{1}\right)} \int_{0}^{h} g_{1}(s) T_{1} v(s) d s\right) \\
& +\frac{1}{\Gamma\left(\alpha-\gamma_{1}\right)} \int_{0}^{t_{1}}\left|\frac{\left(t_{1}-s\right)^{\alpha-1-\gamma_{1}}}{1+t_{1}^{\alpha-1-\gamma_{1}}}-\frac{\left(t_{2}-s\right)^{\alpha-1-\gamma_{1}}}{1+t_{2}^{\alpha-1-\gamma_{1}}}\right|\left|\varphi\left(s, \nu(s), D^{\gamma_{1}} v(s)\right)\right| d s \\
& +\frac{1}{\Gamma\left(\alpha-\gamma_{1}\right)} \int_{t_{1}}^{t_{2}} \frac{\left(t_{2}-s\right)^{\alpha-1-\gamma_{1}}}{1+t_{2}^{\alpha-1-\gamma_{1}}}\left|\varphi\left(s, v(s), D^{\gamma_{1}} v(s)\right)\right| d s \\
& \leq \varepsilon a \sigma_{1}\left(\frac{\Gamma(\alpha)}{\Gamma\left(\alpha-\gamma_{1}\right)}+\frac{2 \omega_{1} l_{1} \Gamma(\alpha-1)}{\Gamma\left(\alpha-\gamma_{1}\right)}\right)+\frac{\varepsilon \sigma_{1} \Gamma(\alpha)}{\Gamma\left(\alpha-\gamma_{1}\right)} \int_{0}^{+\infty}\left|\varphi\left(s, \nu(s), D^{\gamma_{1}} v(s)\right)\right| d s \\
& +\frac{\varepsilon \sigma_{1} M \xi^{\alpha-1}}{\Gamma\left(\alpha-\gamma_{1}\right)} \int_{0}^{\xi}\left|\varphi\left(s, v(s), D^{\gamma_{1}} v(s)\right)\right| d s \\
& +\frac{2 \varepsilon l_{1}\left(\sigma_{1} \omega_{1} M \xi^{\alpha-1}+\delta_{1}\right)}{\Gamma\left(\alpha-\gamma_{1}\right)} \int_{0}^{+\infty}\left|\varphi\left(s, \nu(s), D^{\gamma_{1}} v(s)\right)\right| d s \\
& +\frac{\varepsilon}{\Gamma\left(\alpha-\gamma_{1}\right)} \int_{0}^{t_{1}}\left|\varphi\left(s, v(s), D^{\gamma_{1}} v(s)\right)\right| d s \\
& +\frac{1}{\Gamma\left(\alpha-\gamma_{1}\right)} \int_{t_{1}}^{t_{2}}\left|\varphi\left(s, \nu(s), D^{\gamma_{1}} v(s)\right)\right| d s
\end{aligned}
$$




$$
\begin{aligned}
\leq & \frac{2 \varepsilon a \sigma_{1} \Gamma(\alpha-1)}{\Gamma\left(\alpha-\gamma_{1}\right)}\left(1+\omega_{1} l_{1}\right) \\
& +\left(\varepsilon \zeta_{1}+\frac{1}{\Gamma\left(\alpha-\gamma_{1}\right)}\right) \int_{0}^{+\infty}\left(c_{1}(s)+c_{2}(s)|v(s)|+c_{3}(s)\left|D^{\gamma_{1}} v(s)\right|\right) d s
\end{aligned}
$$

Thus we have

$$
\begin{aligned}
& \left|\frac{T_{1} v\left(t_{2}\right)}{1+t_{2}^{\alpha-1}}-\frac{T_{1} v\left(t_{1}\right)}{1+t_{1}^{\alpha-1}}\right|+\left|\frac{D^{\gamma_{1}} T_{1} v\left(t_{2}\right)}{1+t_{2}^{\alpha-1-\gamma_{1}}}-\frac{D^{\gamma_{1}} T_{1} v\left(t_{1}\right)}{1+t_{1}^{\alpha-1-\gamma_{1}}}\right| \\
& \leq\left(\varepsilon\left(L_{1}+\zeta_{1}\right)+\frac{1}{\Gamma\left(\alpha-\gamma_{1}\right)}+\frac{2 \varepsilon}{\Gamma(\alpha)}\right) \int_{0}^{+\infty}\left(c_{1}(s)+c_{2}(s)|v(s)|+c_{3}(s)\left|D^{\gamma_{1}} v(s)\right|\right) d s \\
& \quad+\varepsilon a \sigma_{1}\left(1+\omega_{1} l_{1}\right)\left(1+\frac{2 \Gamma(\alpha-1)}{\Gamma\left(\alpha-\gamma_{1}\right)}\right) .
\end{aligned}
$$

Then, for all $\varepsilon>0$, there exists $\mu>0$ such that, for $t_{1}, t_{2}>\mu, T_{1}: \omega \rightarrow \omega$ is equiconvergent at infinity. Using the same argument, $T_{2}: \omega \rightarrow \omega$ is also equiconvergent at infinity. Thus $T: \omega \rightarrow \omega$ is equiconvergent at infinity. By means of Remark 2.2, we know $T: B_{R} \rightarrow B_{R}$ is completely continuous.

According to Schauder's fixed point theorem, we conclude that $T$ has at least one fixed point, that is, system (1.1) has at least one solution in $B_{R}$.

\section{Corollary 3.1 Assume that}

$\left(H_{3}\right)$ there exist nonnegative functions $a(t), b(t), a_{i}(t) \in L^{1}(J) \cap C(J), i=1,2$, such that

$$
\begin{aligned}
& |\varphi(t, u, v)| \leq a(t)+a_{1}(t)\left(|u|^{p_{1}}+|v|^{p_{2}}\right), \quad 0<p_{i}<1, i=1,2, t \in J, \\
& \int_{0}^{+\infty} a(t) d t<+\infty, \quad \int_{0}^{+\infty} a_{1}(t) \theta(t) d t<\max \left\{\frac{1}{4 L_{1}}, \frac{1}{4 \zeta_{1}}\right\}
\end{aligned}
$$

and

$$
\begin{aligned}
& |\psi(t, u, v)| \leq b(t)+a_{2}(t)\left(|u|^{q_{1}}+|v|^{q_{2}}\right), \quad 0<q_{i}<1, i=1,2, t \in J, \\
& \int_{0}^{+\infty} b(t) d t<+\infty, \quad \int_{0}^{+\infty} a_{2}(t) \theta(t) d t<\max \left\{\frac{1}{4 L_{2}}, \frac{1}{4 \zeta_{2}}\right\} .
\end{aligned}
$$

Here, $a_{i}, b_{i}, i=1,2$, are nonnegative constants, then system (1.1) has at least one solution.

Proof In this case, let $p *=\max \left\{p_{1}, p_{2}\right\}, q *=\max \left\{q_{1}, q_{2}\right\}$, we take

$$
\begin{aligned}
R> & \left\{\left(\frac{a \sigma_{1}\left(1+\omega_{1} l_{1}\right)+L_{1} \int_{0}^{+\infty} a(s) d s}{\frac{1}{2}-2 L_{1} \int_{0}^{+\infty} a_{1}(s) \theta(s) d s}\right)^{1 / p *},\left(\frac{\frac{2 a \sigma_{1} \Gamma(\alpha-1)}{\Gamma\left(\alpha-\gamma_{1}\right)}\left(1+\omega_{1} l_{1}\right)+\zeta_{1} \int_{0}^{+\infty} a(s) d s}{\frac{1}{2}-2 \zeta_{1} \int_{0}^{+\infty} a_{1}(s) \theta(s) d s}\right)^{1 / p *}\right. \\
& \left(\frac{b \sigma_{2}\left(1+\omega_{2} l_{2}\right)+L_{2} \int_{0}^{+\infty} b(s) d s}{\frac{1}{2}-2 L_{2} \int_{0}^{+\infty} a_{2}(s) \theta(s) d s}\right)^{1 / q *}, \\
& \left.\left(\frac{\frac{2 b \sigma_{2} \Gamma(\beta-1)}{\Gamma\left(\beta-\gamma_{2}\right)}\left(1+\omega_{2} l_{2}\right)+\zeta_{2} \int_{0}^{+\infty} b(s) d s}{\frac{1}{2}-2 \zeta_{2} \int_{0}^{+\infty} a_{2}(s) \theta(s) d s}\right)^{1 / q *}\right\} .
\end{aligned}
$$

The rest of the proof is similar to Theorem 3.1, so we omit the details. 
Remark 3.1 For the sake of simplicity, if $a(t)=b(t)=0$ in condition $\left(H_{3}\right)$, that is,

$$
\begin{aligned}
& |\varphi(t, u, v)| \leq a_{1}(t)\left(|u|^{p_{1}}+|v|^{p_{2}}\right), \quad p_{i}>1, i=1,2, t \in J \\
& \int_{0}^{+\infty} a_{1}(t) \theta(t) d t<\max \left\{\frac{1}{4 L_{1}}, \frac{1}{4 \zeta_{1}}\right\}
\end{aligned}
$$

and

$$
\begin{aligned}
& |\psi(t, u, v)| \leq a_{2}(t)\left(|u|^{q_{1}}+|v|^{q_{2}}\right), \quad q_{i}>1, i=1,2, t \in J, \\
& \int_{0}^{+\infty} a_{2}(t) \theta(t) d t<\max \left\{\frac{1}{4 L_{2}}, \frac{1}{4 \zeta_{2}}\right\} .
\end{aligned}
$$

Due to the different values of $R$, the conclusion of Theorem 3.1 is also true for the nonstrict inequalities $p_{i}, q_{i}>1$. It should be replaced by a weak form which can be derived easily from (3.2) and (3.3).

When $h=0$, the boundary conditions of system (1.1) are changed to the form:

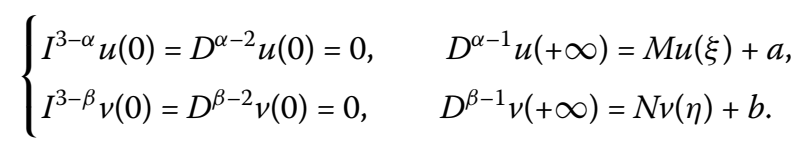

Similar to Theorem 3.1, we can obtain the following result.

\section{Theorem 3.2 Assume that}

$\left(H_{1}^{\prime}\right)$ there exist nonnegative functions $c_{i}(t), d_{i}(t) \in L^{1}(J), i=1,2,3$, such that

$$
\begin{aligned}
& |\varphi(t, u, v)| \leq c_{1}(t)+c_{2}(t)|u|+c_{3}(t)|v|, \quad t \in[0,+\infty), \\
& \int_{0}^{+\infty} c_{1}(t) d t<+\infty, \quad \int_{0}^{+\infty}\left(c_{2}(t)+c_{3}(t)\right) \theta(t) d t<\max \left\{\frac{1}{2 L_{1}^{\prime}}, \frac{1}{2 \zeta_{1}^{\prime}}\right\}
\end{aligned}
$$

where

$$
L_{1}^{\prime}=\sigma_{1}, \quad \zeta_{1}^{\prime}=\frac{1+\sigma_{1}\left(1+M \xi^{\alpha-1}\right)}{\Gamma\left(\alpha-\gamma_{1}\right)}
$$

and

$$
\begin{aligned}
& |\psi(t, u, v)| \leq d_{1}(t)+d_{2}(t)|u|+d_{3}(t)|v|, \quad t \in[0,+\infty), \\
& \int_{0}^{+\infty} d_{1}(t) d t<+\infty, \quad \int_{0}^{+\infty}\left(d_{2}(t)+d_{3}(t)\right) \theta(t) d t<\max \left\{\frac{1}{2 L_{2}^{\prime}}, \frac{1}{2 \zeta_{2}^{\prime}}\right\},
\end{aligned}
$$

where

$$
L_{2}^{\prime}=\sigma_{2}, \quad \zeta_{2}^{\prime}=\frac{1+\sigma_{2}\left(1+N \eta^{\beta-1}\right)}{\Gamma\left(\beta-\gamma_{2}\right)}
$$

Then system (1.1) with boundary condition (3.4) has at least one solution. 
Theorem 3.3 Assume that $\left(H_{1}\right),\left(H_{2}\right)$ hold, then system (1.1) has a unique solution if

$$
m_{1}+m_{2}<1, \quad n_{1}+n_{2}<1
$$

where

$$
\begin{array}{ll}
m_{1}=L_{1} \int_{0}^{+\infty} \lambda_{1}(s) \theta(s) d s, & n_{1}=L_{2} \int_{0}^{+\infty} \lambda_{2}(s) \theta(s) d s, \\
m_{2}=\zeta_{1} \int_{0}^{+\infty} \lambda_{1}(s) \theta(s) d s, & n_{2}=\zeta_{2} \int_{0}^{+\infty} \lambda_{2}(s) \theta(s) d s .
\end{array}
$$

Proof Let $u_{i}(t), v_{i}(t) \in C^{1}(J), i=1,2$; then we have

$$
\begin{aligned}
& \left|\frac{T_{1} v_{2}(t)}{1+t^{\alpha-1}}-\frac{T_{1} v_{1}(t)}{1+t^{\alpha-1}}\right| \\
& \quad \leq \int_{0}^{+\infty} \frac{H(t, s)}{1+t^{\alpha-1}}\left|\varphi\left(s, v_{2}(s), D^{\gamma_{1}} v_{2}(s)\right)-\varphi\left(s, v_{1}(s), D^{\gamma_{1}} v_{1}(s)\right)\right| d s \\
& \quad \leq L_{1} \int_{0}^{+\infty} \lambda_{1}(s) \theta(s) d s\left\|v_{2}-v_{1}\right\|_{X}=m_{1}\left\|v_{2}-v_{1}\right\|_{X}
\end{aligned}
$$

and

$$
\begin{aligned}
& \left|\frac{D^{\gamma_{1}} T_{1} v_{2}(t)}{1+t^{\alpha-1-\gamma_{1}}}-\frac{D^{\gamma_{1}} T_{1} v_{1}(t)}{1+t^{\alpha-1-\gamma_{1}}}\right| \\
& \leq \frac{\sigma_{1} \Gamma(\alpha)}{\Gamma\left(\alpha-\gamma_{1}\right)} \int_{0}^{+\infty}\left|\varphi\left(s, v_{2}(s), D^{\gamma_{1}} v_{2}(s)\right)-\varphi\left(s, v_{1}(s), D^{\gamma_{1}} v_{1}(s)\right)\right| d s \\
& \quad+\frac{M \sigma_{1} \xi^{\alpha-1}}{\Gamma\left(\alpha-\gamma_{1}\right)} \int_{0}^{\xi}\left|\varphi\left(s, v_{2}(s), D^{\gamma_{1}} v_{2}(s)\right)-\varphi\left(s, v_{1}(s), D^{\gamma_{1}} v_{1}(s)\right)\right| d s \\
& \quad+\frac{1}{\Gamma\left(\alpha-\gamma_{1}\right)} \int_{0}^{t}\left|\varphi\left(s, v_{2}(s), D^{\gamma_{1}} v_{2}(s)\right)-\varphi\left(s, v_{1}(s), D^{\gamma_{1}} v_{1}(s)\right)\right| d s \\
& \quad+\frac{2\left(1+\sigma_{1} M \xi^{\alpha-2}\right)}{\Gamma\left(\alpha-\gamma_{1}\right)} \int_{0}^{h} g_{1}(s)\left(T_{1} v_{2}(s)-T_{1} v_{1}(s)\right) d s \\
& \leq \zeta_{1} \int_{0}^{+\infty} \lambda_{1}(s) \theta(s) d s\left\|v_{2}-v_{1}\right\|_{X}=m_{2}\left\|v_{2}-v_{1}\right\|_{X} .
\end{aligned}
$$

We can see that

$$
\left\|T_{1} v_{2}-T_{1} v_{1}\right\|_{X} \leq\left(m_{1}+m_{2}\right)\left\|v_{2}-v_{1}\right\|_{X}
$$

Analogously, it can be proved that

$$
\begin{aligned}
\left|\frac{T_{2} u_{2}(t)}{1+t^{\beta-1}}-\frac{T_{2} u_{1}(t)}{1+t^{\beta-1}}\right| & \leq \sigma_{2}\left(1+\delta_{2} l_{2}\right) \int_{0}^{+\infty} \lambda_{2}(s) \theta(s) d s\left\|u_{2}-u_{1}\right\|_{Y} \\
& =n_{1}\left\|u_{2}-u_{1}\right\|_{Y}
\end{aligned}
$$

and

$$
\left|\frac{D^{\gamma_{2}} T_{2} u_{2}(t)}{1+t^{\beta-1-\gamma_{2}}}-\frac{D^{\gamma_{2}} T_{2} u_{1}(t)}{1+t^{\beta-1-\gamma_{2}}}\right|
$$




$$
\begin{aligned}
\leq & \left(\frac{\sigma_{1}\left(\Gamma(\alpha)+M \xi^{\alpha-1}\right)+1}{\Gamma\left(\alpha-\gamma_{1}\right)}+\frac{\left(\sigma_{1} \omega_{1} M \xi^{\alpha-1}+\delta_{1}\right)}{\left(1-\mu_{1}\right) \Gamma(\alpha)} \cdot \frac{2\left(1+\sigma_{1} M \xi^{\alpha-2}\right)}{\Gamma\left(\alpha-\gamma_{1}\right)}\right) \\
& \times \int_{0}^{+\infty} \lambda_{2}(s) \theta(s) d s\left\|u_{2}-u_{1}\right\|_{Y} \\
= & n_{2}\left\|u_{2}-u_{1}\right\|_{Y} .
\end{aligned}
$$

Thus we know that

$$
\left\|T_{2} u_{2}-T_{2} u_{1}\right\|_{Y} \leq\left(n_{1}+n_{2}\right)\left\|u_{2}-u_{1}\right\|_{Y}
$$

In conclusion, we have

$$
\left\|T\left(u_{2}, v_{2}\right)-T\left(u_{1}, v_{1}\right)\right\|_{Q} \leq \max \left\{m_{1}+m_{2}, n_{1}+n_{2}\right\}\left\|\left(u_{2}, v_{2}\right)-\left(u_{1}, v_{1}\right)\right\|_{Q} .
$$

Obviously, $T$ is a contraction. By means of the Banach contraction theorem, $T$ has a unique fixed point which is the unique solution of system (1.1).

Corollary 3.2 Assume that $\left(H_{2}\right),\left(H_{3}\right)$ hold, then system (1.1) has a unique solution if $m_{1}+$ $m_{2}<1, n_{1}+n_{2}<1$.

Corollary 3.3 On the basis of Remark 3.1, if condition $\left(H_{2}\right)$ holds, then system (1.1) has a unique solution if $m_{1}+m_{2}<1, n_{1}+n_{2}<1$. In short, if $\varphi$, $\psi$ are bounded and continuous on $J \times R \times R$, then there exists a solution for system (1.1).

Remark 3.2 If $\varphi, \psi \in C\left(J \times \mathbb{R}^{+} \times \mathbb{R}^{+}, \mathbb{R}^{+}\right), \varphi(t, u, v), \psi(t, u, v) \not \equiv 0$, under condition $\left(H_{1}\right)$ or $\left(H_{3}\right)$, then system (1.1) has at least one positive solution. Further, the positive solution is unique if $\left(H_{1}\right),\left(H_{2}\right)$ or $\left(H_{2}\right),\left(H_{3}\right)$ are satisfied with $m_{1}+m_{2}<1, n_{1}+n_{2}<1$.

\section{An example}

Example 4.1 Consider the system

$$
\left\{\begin{array}{l}
D^{\frac{5}{2}} u(t)+\frac{\sin t}{\sqrt{2+t^{2}}}+\frac{\sqrt{\left|v(t) D^{\frac{1}{2}} v(t)\right|}}{72 e^{t}\left(1+t^{\frac{3}{2}}\right)}=0, \quad t \in[0,+\infty), \\
D^{\frac{5}{2}} v(t)+(2+\cos t) e^{-t^{2}}+\frac{\sin |u(t)|}{48 e^{t}\left(1+t^{\frac{3}{2}}\right)}+\frac{\ln \left(1+\left|D^{\frac{1}{2}} u(t)\right|\right)}{192 e^{\sqrt{t}}\left(1+t^{\frac{3}{2}}\right)}=0, \\
I^{\frac{1}{2}} u(0)=0, \quad D^{\frac{1}{2}} u(0)=\left(\frac{3 \pi^{2}}{16}-\frac{\pi}{4}\right) \int_{0}^{1} s^{4} u(s) d s, \quad D^{\frac{3}{2}} u(+\infty)=u(1)+2, \\
I^{\frac{1}{2}} v(0)=0, \quad D^{\frac{1}{2}} v(0)=\left(\frac{\pi^{2}}{8}-\frac{\pi}{6}\right) \int_{0}^{1} s^{2} v(s) d s, \quad D^{\frac{3}{2}} v(+\infty)=v(1)+2,
\end{array}\right.
$$

where $\alpha=\beta=\frac{5}{2}, \gamma_{1}=\gamma_{2}=\frac{1}{2}, h=1, M, N, \xi, \eta=1, a=b=2, g_{1}(t)=\left(\frac{3 \pi^{\frac{3}{2}}}{16}-\frac{\pi}{4}\right) t^{4}, g_{2}(t)=$ $\left(\frac{\pi^{\frac{3}{2}}}{8}-\frac{\pi}{6}\right) t^{2}$, and

$$
\begin{aligned}
& \varphi(t, u, v)=\frac{\sin t}{\sqrt{2+t^{2}}}+\frac{\sqrt{|u v|}}{72 e^{t}\left(1+t^{\frac{3}{2}}\right)} \\
& \psi(t, u, v)=(2+\cos t) e^{-t^{2}}+\frac{\sin |u|}{48 e^{t}\left(1+t^{\frac{3}{2}}\right)}+\frac{\ln (1+|v|)}{192 e^{\sqrt{t}}\left(1+t^{\frac{3}{2}}\right)} .
\end{aligned}
$$


Choose

$$
\begin{aligned}
& c_{1}(t)=\frac{1}{\sqrt{2+t^{2}}}, \quad c_{2}(t)=c_{3}(t)=\frac{1}{144 e^{t}\left(1+t^{\frac{3}{2}}\right)} \\
& d_{1}(t)=3 e^{-t^{2}}, \quad d_{2}(t)=\frac{1}{48 e^{t}\left(1+t^{\frac{3}{2}}\right)}, \quad d_{3}(t)=\frac{1}{192 e^{\sqrt{t}}\left(1+t^{\frac{3}{2}}\right)} .
\end{aligned}
$$

Obviously, $|\varphi(t, u, v)| \leq c_{1}(t)+c_{2}(t)|u|+c_{3}(t)|v|,|\psi(t, u, v)| \leq d_{1}(t)+d_{2}(t)|u|+d_{3}(t)|v|$, and by simple computations, we find that $0<M \xi^{\alpha-1}, N \eta^{\beta-1}<\Gamma\left(\frac{5}{2}\right) \approx 1.329, \sigma_{1}=\sigma_{2}=$ $\frac{4}{3 \sqrt{\pi}-4}, T_{1}(t)=T_{2}(t)=\frac{8}{3 \pi-4 \sqrt{\pi}} t^{\frac{3}{2}}+\frac{2}{\sqrt{\pi}} t^{\frac{1}{2}}, \mu_{1}=\int_{0}^{1} g_{1}(t) T_{1}(t) d t=\frac{\sqrt{\pi}}{14}+\frac{3 \pi-4 \sqrt{\pi}}{24}<1, \mu_{2}=$ $\int_{0}^{1} g_{2}(t) T_{2}(t) d t=\frac{\pi}{8}-\frac{\sqrt{\pi}}{12}<1, \delta_{1}=\frac{9 \pi^{\frac{3}{2}}-12 \pi}{140}, \delta_{2}=\frac{7 \pi^{\frac{3}{2}}}{96}-\frac{7 \pi}{72}, \omega_{1}=\frac{3 \pi^{\frac{3}{2}}-4 \pi}{112}, \omega_{2}=\frac{\pi^{\frac{3}{2}}}{32}-\frac{\pi}{24}$, $l_{1}=\frac{1008}{(3 \sqrt{\pi}-4)(168+16 \sqrt{\pi}-21 \pi)}$ and $l_{2}=\frac{144}{(3 \sqrt{\pi}-4)(24+2 \sqrt{\pi}-3 \pi)}$. Further, we can obtain

$$
\begin{aligned}
\int_{0}^{+\infty} c_{1}(t) d t=\frac{\pi}{4}<+\infty & \\
\int_{0}^{+\infty}\left(c_{2}(t)+c_{3}(t)\right) \theta(t) d t & =\frac{1}{72} \int_{0}^{1} \frac{1+t}{e^{t}\left(1+t^{\frac{3}{2}}\right)} d t+\frac{1}{72} \int_{1}^{+\infty} e^{-t} d t \\
& \leq \frac{1}{72}\left(2+\frac{1}{e}-\frac{4 \ln 2}{3}\right)<\max \left\{\frac{1}{2 L_{1}}, \frac{1}{2 \zeta_{1}}\right\}
\end{aligned}
$$

Here,

$$
\begin{aligned}
& L_{1}=\sigma_{1}\left(1+\delta_{1} l_{1}\right)=\frac{4}{3 \sqrt{\pi}-4}+\frac{3024 \pi}{35(3 \sqrt{\pi}-4)(168+16 \sqrt{\pi}-21 \pi)} \approx 4.617, \\
& \begin{aligned}
\zeta_{1}=\frac{6 \sqrt{\pi}}{3 \sqrt{\pi}-4}+\frac{4536 \pi^{\frac{3}{2}}-1008 \pi}{35(3 \sqrt{\pi}-4)(168+16 \sqrt{\pi}-21 \pi)} \approx 11.747, \\
\int_{0}^{+\infty} d_{1}(t) d t=\frac{3 \sqrt{\pi}}{2}<+\infty, \\
\int_{0}^{+\infty}\left(d_{2}(t)+d_{3}(t)\right) \theta(t) d t=\int_{0}^{1}\left(\frac{1+t}{48 e^{t}\left(1+t^{\frac{3}{2}}\right)}+\frac{1+t}{192 e^{\sqrt{t}}\left(1+t^{\frac{3}{2}}\right)}\right) d t \\
\quad \leq \frac{5}{96}-\frac{5 \ln 2}{144}+\frac{1}{32 e}<\max \left\{\frac{1}{2 L_{2}}, \frac{1}{2 \zeta_{2}}\right\} \\
L_{2}=\sigma_{2}\left(1+\delta_{2} l_{2}\right)=\frac{4}{3 \sqrt{\pi}-4}+\frac{1}{(3 \sqrt{\pi}-4)(24+2 \sqrt{\pi}-3 \pi)} \approx 4.879,
\end{aligned}
\end{aligned}
$$

and

$$
\zeta_{2}=\frac{6 \sqrt{\pi}}{3 \sqrt{\pi}-4}+\frac{21 \pi^{\frac{3}{2}}-7 \pi}{(3 \sqrt{\pi}-4)(24+2 \sqrt{\pi}-3 \pi)} \approx 12.050 .
$$

Then the conditions of Theorem 3.1 are satisfied, so system (4.1) has at least one solution. 


\section{Funding}

This paper was supported financially by the Youth Science Foundation of China (11201272) and Shanxi Province Science Foundation (2015011005).

Availability of data and materials

Not applicable.

\section{Competing interests}

The authors declare that they have no competing interests.

\section{Authors' contributions}

The authors declare that the study was realized in collaboration with the same responsibility. All authors read and approved the final manuscript.

\section{Publisher's Note}

Springer Nature remains neutral with regard to jurisdictional claims in published maps and institutional affiliations.

Received: 20 August 2018 Accepted: 19 June 2019 Published online: 05 July 2019

References

1. Kilbas, A., Srivastava, H., Trujillo, J.: Theory and Applications of Fractional Differential Equations. Elsevier, Amsterdam (2006)

2. Lakshmikantham, V., Leela, S., Devi, J.V.: Theory of Fractional Dynamic Systems. Cambridge Academic Publishers, Cambridge (2009)

3. Podlubny, I.: Fractional Differential Equations. Academic Press, San Diego (1999)

4. Ahmad, B., Luca, R.: Existence of solutions for a system of fractional differential equations with coupled nonlocal boundary conditions. Fract. Calc. Appl. Anal. 21(2), 423-441 (2018)

5. Bai, Z.: Eigenvalue intervals for a class of fractional boundary value problem. Comput. Math. Appl. 64(10), 3253-3257 (2012)

6. Cui, Y., Ma, W., Sun, Q., Su, X.: New uniqueness results for boundary value problem of fractional differential equation. Nonlinear Anal., Model. Control 23(1), 31-39 (2018)

7. Graef, J.R., Kong, L., Kong, Q., Wang, M.: Uniqueness of positive solutions of fractional boundary value problems with non-homogeneous integral boundary conditions. Fract. Calc. Appl. Anal. 15(3), 509-528 (2012)

8. Wang, J., Zhou, Y.: A class of fractional evolution equations and optimal controls. Nonlinear Anal., Real World Appl. $12(1), 262-272(2011)$

9. Xu, N., Liu, W.: Iterative solutions for a coupled system of fractional differential-integral equations with two-point boundary conditions. Appl. Math. Comput. 244, 903-911 (2014)

10. Liu, L., Sun, F., Zhang, X., Wu, Y.: Bifurcation analysis for a singular differential system with two parameters via to degree theory. Nonlinear Anal., Model. Control 22(1), 31-50 (2017)

11. Ren, J., Zhai, C.: A fractional $q$-difference equation with integral boundary conditions and comparison theorem. Int. J. Nonlinear Sci. Numer. Simul. 18(7-8), 575-583 (2017)

12. Zhai, C., Ren, J.: The unique solution for a fractional $q$-difference equation with three-point boundary conditions. Indag. Math. 29, 948-961 (2018)

13. Trif, T.: Existence of solutions to initial value problems for nonlinear fractional differential equations on the semi-axis. Fract. Calc. Appl. Anal. 3, 595-612 (2013)

14. Wang, J., Fečkan, M., Zhou, Y.: A survey on impulsive fractional differential equations. Fract. Calc. Appl. Anal. 1, 806-831 (2016)

15. Wang, W., Guo, X.: Eigenvalue problem for fractional differential equations with nonlinear integral and disturbance parameter in boundary conditions. Bound. Value Probl. 2016, 42 (2016)

16. Zhai, C., Ren, J.: Some properties of sets, fixed point theorems in ordered product spaces and applications to a nonlinear system of fractional differential equations. Topol. Methods Nonlinear Anal. 49, 625-645 (2017)

17. Zhai, C., Jiang, R.: Unique solutions for a new coupled system of fractional differential equations. Adv. Differ. Equ. 2018, 1 (2018)

18. Zhang, H., Li, Y., Lu, W.: Existence and uniqueness of solutions for a coupled system of nonlinear fractional differential equations with fractional integral boundary conditions. J. Nonlinear Sci. Appl. 9, 2434-2447 (2016)

19. Cui, Y.: Uniqueness of solution for boundary value problems for fractional differential equations. Appl. Math. Lett. 51 48-54 (2016)

20. Graef, J.R., Kong, L., Kong, Q., Wang, M.: Positive solutions of nonlocal fractional boundary value problems. Discrete Contin. Dyn. Syst. 7(4), 283-290 (2013)

21. Guan, Y., Zhao, Z., Lin, X.: On the existence of positive solutions and negative solutions of singular fractional differential equations via global bifurcation techniques. Bound. Value Probl. 2016, 141 (2016)

22. Guo, L., Liu, L., Wu, Y.: Existence of positive solutions for singular higher-order fractional differential equations with infinite-points boundary conditions. Bound. Value Probl. 2016, 114 (2016)

23. Henderson, J., Luca, R.: Existence of nonnegative solutions for a fractional integro-differential equation. Results Math. 72, 747-763 (2017)

24. Marasi, H.R., Afshari, H., Zhai, C.: Some existence and uniqueness results for nonlinear fractional partial differential equations. Rocky Mt. J. Math. 47(2), 571-585 (2017)

25. Wang, W., Guo, X.: Eigenvalue problem for fractional differential equations with nonlinear integral and disturbance parameter in boundary conditions. Bound. Value Probl. 2016, 42 (2016)

26. Wang, H., Zhang, L.: The solution for a class of sum operator equation and its application to fractional differential equation boundary value problems. Bound. Value Probl. 2015, 203 (2015) 
27. Wang, Y., Liu, L.: Uniqueness and existence of positive solutions for the fractional integro-differential equation. Bound. Value Probl. 2017, 12 (2017)

28. Zhai, C., Yan, W., Yang, C.: A sum operator method for the existence and uniqueness of positive solutions to Riemann-Liouville fractional differential equation boundary value problems. Commun. Nonlinear Sci. Numer. Simul. $18,858-866$ (2013)

29. Zhai, C., Xu, L.: Properties of positive solutions to a class of four-point boundary value problem of Caputo fractional differential equations with a parameter. Commun. Nonlinear Sci. Numer. Simul. 19, 2820-2827 (2014)

30. Zhang, K.: On sign-changing solution for some fractional differential equations. Bound. Value Probl. 2017, 59 (2017)

31. Ali, A., Shah, K., Khan, R.A.: Existence of positive solution to a class of boundary value problems of fractional differential equations. Comput. Methods Differ. Equ. 4, 19-29 (2016)

32. Liu, S., Li, H.: Extremal system of solutions for a coupled system of nonlinear fractional differential equations by monotone iterative method. J. Nonlinear Sci. Appl. 9, 3310-3318 (2016)

33. Su, X.: Boundary value problem for a coupled system of nonlinear fractional differential equations. Appl. Math. Lett. 22, 64-69 (2009)

34. Wang, G., Pei, K., Agarwal, R., Zhang, L., Ahmad, B.: Nonlocal Hadamard fractional boundary value problem with Hadamard integral and discrete boundary conditions on a half-line. J. Comput. Appl. Math. 342, 230-239 (2018)

35. Li, X., Liu, X., Jia, M., Li, Y., Zhang, S.: Existence of positive solutions for integral boundary value problems of fractional differential equations on infinite interval. Math. Methods Appl. Sci. 40, 1892-1904 (2017)

36. Cerdik, T.S., Deren, F.Y., Hamal, N.A.: Unbounded solutions for boundary value problems of Riemann Liouville fractional differential equations on the half-line. Fixed Point Theory 19, 93-106 (2018)

37. Kou, C., Zhou, H., Yan, Y.: Existence of solutions of initial value problems for nonlinear fractional differential equations on the half-axis. Nonlinear Anal. 74, 5975-5986 (2011)

38. Thiramanus, P., Ntouyas, S.K., Tariboon, J.: Positive solutions for Hadamard fractional differential equations on infinite domain. Adv. Differ. Equ. 2016, 1 (2016)

39. Zhang, L., Ahmad, B., Wang, G.: Monotone iterative method for a class of nonlinear fractional differential equations on unbounded domains in Banach spaces. Filomat 31, 1331-1338 (2017)

40. Pei, K., Wang, G., Sun, Y.: Successive iterations and positive extremal solutions for a Hadamard type fractional integro-differential equations on infinite domain. Appl. Math. Comput. 312, 158-168 (2017)

41. Su, X., Zhang, S.: Unbounded solutions to a boundary value problem of fractional order on the half-line. Comput. Math. Appl. 61, 1079-1087 (2011)

42. Arara, A., Benchohra, M., Hamidi, N., Nieto, J.J.: Fractional order differential equations on an unbounded domain. Nonlinear Anal. 72, 580-586 (2010)

43. Liang, S., Zhang, J.: Existence of three positive solutions of $m$-point boundary value problems for some nonlinear fractional differential equations on an infinite interval. Comput. Math. Appl. 61, 3343-3354 (2011)

44. Tariboon, J., Ntouyas, S.K., Asawasamrit, S., Promsakon, C.: Positive solutions for Hadamard differential systems with fractional integral conditions on an unbounded domain. Open Math. 15, 645-666 (2017)

45. Wang, G., Ahmad, B., Zhang, L.: A coupled system of nonlinear fractional differential equations with multipoint fractional boundary conditions on an unbounded domain. Abstr. Appl. Anal. 2, 1-11 (2012)

46. Zhai, C., Wang, W. Properties of positive solutions for $m$-point fractional differential equations on an infinite interval. Rev. R. Acad. Cienc. Exactas Fís. Nat., Ser. A Mat. 113, 1289-1298 (2019)

47. Zhang, X:: Positive solutions for a class of singular fractional differential equation with infinite-point boundary value conditions. Appl. Math. Lett. 39, 22-27 (2015)

\section{Submit your manuscript to a SpringerOpen ${ }^{\circ}$ journal and benefit from:}

- Convenient online submission

- Rigorous peer review

- Open access: articles freely available online

- High visibility within the field

- Retaining the copyright to your article

Submit your next manuscript at $\gg$ springeropen.com 